This item was submitted to Loughborough's Research Repository by the author.

Items in Figshare are protected by copyright, with all rights reserved, unless otherwise indicated.

\title{
X-ray micro-computed tomography $(\mu-C T)$ for 3D characterization of particle kinematics representing water-induced loess micro-fabric collapse
}

PLEASE CITE THE PUBLISHED VERSION

https://doi.org/10.1016/j.enggeo.2020.105895

\section{PUBLISHER}

Elsevier BV

VERSION

AM (Accepted Manuscript)

\section{PUBLISHER STATEMENT}

This paper was accepted for publication in the journal Engineering Geology and the definitive published version is available at https://doi.org/10.1016/j.enggeo.2020.105895

LICENCE

CC BY-NC-ND 4.0

\section{REPOSITORY RECORD}

Yu, B, W Fan, JH Fan, Tom Dijkstra, YN Wei, and TT Wei. 2020. "X-ray Micro-computed Tomography ( $\mu$-ct) for 3D Characterization of Particle Kinematics Representing Water-induced Loess Micro-fabric Collapse". Loughborough University. https://hdl.handle.net/2134/13274546.v1. 

representing water-induced loess micro-fabric collapse

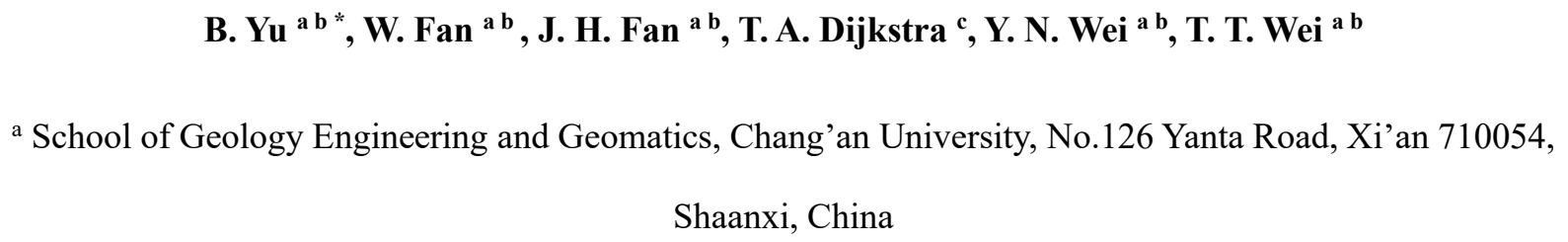

${ }^{\mathrm{b}}$ Key Laboratory of Western China's Mineral Resources and Geological Engineering, Ministry of Education, No.126 Yanta Road, Xi'an 710054, Shaanxi, China

${ }^{c}$ School of Architecture, Building and Civil Engineering, Loughborough University, LE11 3TU, UK

* Corresponding author. Email address: bo.yu@chd.edu.cn (B. Yu)

\section{Abstract}

The 3D characterisation of particle kinematics representing the changes in fabric observed during water-driven collapse of loess is, to the best of our knowledge, performed for the first time at the micron scale. An apparatus is specially designed to perform collapse tests on loess specimens of several millimeters in size and to capture, using X-ray tomography, particle-scale microfabric features of the sample in initial, loaded and flooded (i.e. collapsed) states. Although similar approaches have been used to assess problems such as microstructural evolution in sand, the application of these methods to assess loess collapse behaviour at the micron scale constitutes the novelty of this work. Individual particles within the specimen in the initial and deformed configurations are identified and tracked through an iterative segmentation technique and the particle tracking method 'ID-track'. This allowed determination of particle displacement and rotation. The displacement field within the collapsed sample is found to be less uniform compared to that within the unwetted sample under loading. Coefficient of heterogeneity is defined to quantify the level of non-uniformity of the particle displacements within the deformed sample, revealing higher heterogeneity in the deformation of the collapsed sample compared to that of the unwetted sample under loading. It is also found that particles tend to rotate around axes perpendicular to, rather than parallel to, the direction of major principal stress, and the rotation magnitudes appears to be controlled largely by the size of particles while their morphology plays a minor role. The influence of porosity on the collapse process is quantified significant heterogeneous volumetric strains are observed at the single particle scale. It is also shown that the evolution of particle-to-particle contacts is much more complex than previously stated. The micron-scale investigations of individual particle kinematics following loading and wetting offer exciting new avenues for visualization and an enhanced capability for quantification of loess collapse processes. 


\section{Keywords:}

Loess; Water-induced collapse; Microfabric; Micro-computed tomography; Particle tracking

\section{Introduction}

Loess is an aeolian deposit typically dominated by silt-sized quartz particles, and is distributed across extensive areas of the world, among which the $440,000 \mathrm{~km}^{2}$ Chinese Loess Plateau is known uniquely to possess loess occurrence in thickness of over $300 \mathrm{~m}$. From the engineering point of view, loess is a problem soil with the tendency to collapse when subjected to loading and wetting. When saturated or even unsaturated moisture content levels are attained, a rapid and considerable reduction of strength takes place. The collapse of loess can give rise to many types of geo-hazards, and in turn this can result in severe damages to buildings and infrastructure, threatening people's lives and livelihoods (Juang et al., 2019; Peng et al., 2018).

Relevance between microscale fabric features and macroscale collapse behaviour has been observed for loess deposits across different regions (Derbyshire and Mellors, 1988; Gao, 1981; Lei, 1989; Cui et al., 2004). The mechanism of loess collapsibility lies in the intrinsic open metastable fabric and inter-particle bond structures which can be greatly weakened by water, causing considerable loss of material strength upon wetting (Barden et al., 1973; Yang, 1988; Luo et al., 2018; Shao et al., 2018; Wang et al., 2020). The collapse process can be characterized by the transformation from the initial open particle packing to closer ones at the single particle level (Dijkstra et al., 1995; referred to as hydro-consolidation by Rogers et al., 1994). At the microscale, qualitative observations as well as quantitative analysis on loess microfabric have deepened the understanding of the collapse mechanism, largely with the help of tools such as scanning electron microscope (SEM) and mercury intrusion porosimetry (MIP) (Jiang et al., 2014; Xu and Coop, 2016; Ng et al., 2016; Luo et al., 2018; Wang et al., 2019). Important contributing factors have been highlighted and various conceptual models have been postulated to explain the causes and processes of hydro-consolidation (Liu et al., 2015; Li and Li, 2017). However, although experimental evidence has confirmed that loess with lower dry density (i.e. higher void ratio) has a greater tendency to collapse under loading and wetting, whether the same statement remains true at the scales of individual particles is not yet experimentally demonstrated. In other words, it is not clear whether a locally larger void space in the particle-scale microfabrics will necessarily result in larger deformation during hydro-consolidation. This very issue is difficult to handle in the context of SEM or MIP essentially because it is impossible to follow the spatial trajectory of individual particles or the temporal evolution of local microfabrics due to the destructive nature of these approaches. This is probably also responsible for the lack of progress in the field of loess packing research (Assadi-Langroudi et al., 2018).

In recent years, high resolution X-ray micro computed tomography ( $\mu$-CT) witnessed rapid development 
(Viggiani and Tengattini, 2019; Cnudde and Boone, 2013), which might provide an opportunity to assess the abovementioned problem. X-ray micro tomography is non-destructive and typically provides spatial resolutions ranging from several tens of microns to sub-microns, allowing the particle-scale microstructural features within the same specimen in different loading states to be characterized. Reconstructed images from $\mu$-CT scan can then be dealt with using image processing techniques (e.g. filtering, binarization, segmentation, etc.) in order to quantitatively characterize particle morphology and microfabric in soils (Fonseca et al., 2013b; Fonseca et al., 2013a). With reference to shear response of sand for example, the shear band formation as well as 3D particle displacements have been captured based on $\mu$-CT techniques (Hall et al., 2010; Watanabe et al., 2012). Ando et al. (2012) proposed an 'ID-track' approach to identify individual sand particles within the sample throughout loading based on their morphological features, allowing the particle kinematics (displacements and rotations) to be analyzed. Evolving features such as particle breakage or inter-particle contact evolution have also been investigated with the help of $\mu$ CT scanning and appropriate image processing techniques (Cheng and Wang, 2018). Recently, based on $\mu$-CT, Kong and Fonseca (2018 and 2019) carried out 3D quantitative characterization on the particle morphology of shelly carbonate sand, which is highly irregular in shape, and on the particle kinematics during compression, demonstrating the effectiveness and potential of this approach in analyzing soils with complicated microfabrics. With reference to loess, the authors have already employed the $\mu$-CT technique to characterize some microscopic features in dry and wetted states (Wei et al., 2019; Wei et al., 2020), but the dynamic aspect of the collapse process has not been yet addressed.

Although powerful as tools, the experimental set-ups used in the above-mentioned studies dealing essentially with sands are not readily adoptable to study loess since much smaller specimen size is required in order to achieve sufficient spatial resolution ( $1 \mu \mathrm{m}$ in this study) for loess microfabric characterization. Moreover, the clay-sized particles within loess are basically indistinguishable under common resolutions of $\mu$-CT, and fine particles flocculating at contact areas also pose important difficulties to effective image processing. Taking these issues into account, an experimental apparatus capable to carry out collapse tests on loess samples several millimeters in size is designed, and an iterative segmentation technique is employed in order to make the most of the acquired data. It is the objective of this paper to characterize qualitatively and quantitatively, based on X-ray tomography and image processing techniques, the particle kinematics and microfabric evolution during the process of loess hydroconsolidation, and to provide insights towards an improved understanding of the mechanisms of loess collapsibility.

\section{Experimental methods}

\subsection{Materials description and sample preparation}

The loess sample is collected from an exposed location $2 \mathrm{~m}$ from the upper surface of a cut slope at Yan'an in 
Shaanxi Province, northern China. The material is a typical Late Pleistocene loess which is referred to as 'Malan Loess' in China and shows significant collapsibility when loaded and wetted. In its natural state, the void ratio is 0.83 and the bulk density is $1.71 \mathrm{~g} / \mathrm{cm}^{3}$, with a moisture content of $15 \%$. The particle size distribution obtained through laser diffraction analysis (Fig. 1(a)) exhibits a typical bimodal feature with mean particle size $D_{50}=23 \mu \mathrm{m}$. Angular and subangular silt particles are dominant and exist primarily as skeleton particles that constitutes the highly porous loess microfabric, whereas clay particles have a total percentage of $7.41 \%$, forming the bonding structures or adhering to surfaces of silt particles in a scattered manner or as coatings (see Fig.1(b)). See Wei et al. (2020) for more details of the sampling site and material properties.

The particle-scale observation of loess microfabric based on $\mu$-CT requires the sample to be very small in size (around several millimeters). In this work, the scanned sample is a cylinder $2.7 \mathrm{~mm}$ in diameter and $5 \mathrm{~mm}$ in height, allowing a spatial resolution of $1 \mu \mathrm{m}$ to be achieved. The natural state loess is sampled into a $1 \mathrm{~mm}$ thick polymethyl methacrylate (PMMA) tube which acted as a confining ring during the one-dimensional collapse test. The sampling process calls for extreme care and is described in the following sections.

The loess was firstly sampled into a metallic confining ring with inner diameter and height of $80 \mathrm{~mm}$ and 20 $\mathrm{mm}$, respectively, i.e. similar dimensions of a sample for a conventional laboratory oedometer test. Subsequently, the PMMA tube, inner diameter of $2.7 \mathrm{~mm}$ and height of $5 \mathrm{~mm}$, was perpendicularly inserted into the centre of and perpendicularly indented into the centre of the loess sample. The indenting end of the tube had been sharpened to facilitate the tube to penetrate into the loess. Then the tube, containing the loess sample, was carefully taken out from the surrounding material and was fitted into the apparatus for the micro-collapse test. This sampling process inevitably causes some disturbance of the loess fabric. Prior to the collapse test, a fast tomography scan at low resolution was carried out to check the integrity of the sample and only a small amount of densification and fabric damage was observed along the soil-tube interface. Hence, there is confidence that the characteristic meta-stable loess microfabric remains well preserved throughout the sampling process. This procedure therefore allows the key physical processes at the particle scale during collapse to be characterized based on the micro-collapse test and $\mu$ CT.

\subsection{Experimental set-up and test programme}

An experimental apparatus is designed and constructed to carry out one-dimensional collapse test on very small (2.7 mm diameter, $5 \mathrm{~mm}$ high) loess samples (see Fig. 2(a)). The key parts of the set-up, as shown in Fig. 2(b), are built on an aluminum base plate which can be moved vertically along a threaded guide bar. In order to apply a specific load on the sample, the base plate is displaced upwards and the displacement is monitored by an optical transducer. The sample is loaded and thus compressed once the top loading plate touches the load transducer above. 
The PMMA tube is much stiffer compared to loess and the deformation within the tube during the test is negligible. The contact interface between the confining tube and the loading plate is greased using Vaseline to minimize friction. To flood the sample during the test, water is introduced into the watering chamber inside the base plate. The top end of the sample is lower than the free water surface in the reservoir, allowing water to flow up through the porous stone plate (covered by a sheet of filter paper on its top) and to saturate the sample.

During the $\mu$-CT scan, the sample needs to be positioned very close (about a few millimeters) to the X-ray source lens in order to achieve the expected spatial resolution. Therefore, once reaching the prescribed states (i.e., loaded and flooded) for the scan, the sample must be unloaded and taken out (along with the confining tube) from the apparatus which is too big to be fitted into the scan chamber. This means that only permanent deformation remains within the material. The very small sample size ensures that effects of gravity and kinematic forces are

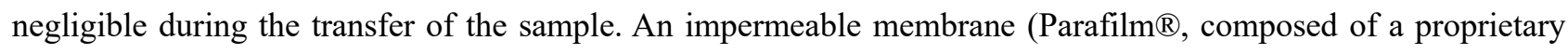
blend of waxes and polyolefins) is wrapped outside the confining tube to minimize moisture loss during the scan.

Figure 3 shows the load-displacement response of the loess sample during the test, with the specific states for $\mu$-CT scan marked as initial, loaded and flooded (collapsed) states. As the first step, the specimen in initial state is scanned prior to the test in order to obtain data of the undeformed microfabric. Being installed back to the apparatus after the first scan, the sample is loaded slowly (at a strain rate of approximately $10^{-4} \mathrm{~min}^{-1}$ ) up to a pressure of 800 $\mathrm{kPa}$ and then unloaded for performing the second scan, which aims at capturing the effect of loading on the microfabric. This pressure level is much higher than that which loess is likely to encounter in reality, aiming at better capture and visualization of the microfabric change due to the collapse. Subsequently, the sample is reloaded again to $800 \mathrm{kPa}$ and flooded to saturation, which causes a sudden drop of the measured pressure. Then, the sample is further compressed at the same rate as previous until the load level of $800 \mathrm{kPa}$ is re-established. The deformation of the sample after flooding corresponds to the amount of wetting-induced collapse. The sample is then unloaded and is subject to the third scan from which images of the collapsed sample are obtained.

It is worth mentioning that the primary function of the set-up in this work is to identify the three characteristic states of the sample during the test for the $\mu$-CT scan, rather than to accurately measure the load and deformation response. The shortening of the sample can be calibrated with the help of the tomography images. The slopes of the loading/unloading curves, which are considerably influenced by the overall stiffness of the apparatus, have little influence on the following microfabric analysis.

\subsection{X-ray micro tomography}

The X-ray tomography scans are performed using Zeiss-Xradia-520-Versa 3D X-ray microscope, which 
provides spatial resolutions as high as $0.7 \mu \mathrm{m}$ thanks to its two-stage magnification technology, at the Core Labs of Institute of Geology and Geophysics of Chinese Academy of Sciences (IGGCAS) in Beijing. The sample to be scanned is fixed on top end of a vertical bar which spins along with the rotating stage during the scan, see Fig. 4(a). For each prescribed state (i.e. initial, loaded and flooded), a full scan of the entire sample as well as a partial scan are carried out as illustrated in Fig. 4(b), with acquisition time being $15 \mathrm{~min}$ and $2 \mathrm{hr}$ respectively. The size of a voxel (basic volumetric unit) for the full scan is $5 \times 5 \times 5 \mu \mathrm{m}^{3}$ while for the partial scan, performed on a cylindrical region of interest (ROI) with $1 \mathrm{~mm}$ both in diameter and in height, the voxel size is $1 \times 1 \times 1 \mu \mathrm{m}^{3}$. The primary purpose of the full scan is to evaluate the integrity of the sample and to select an ideal ROI for the partial scan. Disturbance at the sample-tube interface could be seen (see the snapshot in Fig. 4(b)), which is possibly caused by the sample preparation process. Nevertheless, the majority throughout the sample volume is practically undisturbed, from which a reasonable ROI can be selected. The spatial resolution for the partial scan allows identifying most skeleton particles, the kinematics of which during deformation will be quantitatively characterized and analysed in the following sections.

\section{Image processing}

Image processing, involving a sequence of processes, is essential for identifying and characterizing features of interest based on tomography images. The images obtained from $\mu$-CT were processed using Avizo, a 3D visualization and analysis software that is equipped with mainstream image processing modules and allows also user-defined operations. The sequential steps of image processing followed in this study are illustrated as a flowchart as shown in Fig. 5. Note that the test is not in-situ, hence the image volumes for the sample scanned in different states are aligned before processed, by translations and rotations, to facilitate the analysis of particle kinematics in the same coordinate system. In this study, image processing is performed on a block-shaped volume having dimension of $695 \times 626 \times 767 \mu \mathrm{m}^{3}$ for each state (initial, loaded and collapsed).

In the first step, the 3D grayscale image volume is 'binarized' so that the solid phase (i.e. particles) could be separated from the void space. Binarization resulted in images in which voxels within the solid phase have values of 1 and those within the void space have values of 0 . This is achieved by setting a global threshold value for the grayscale tomography images, in which voxels having values greater than this threshold would be recognized as 'solid'. Ostu's method (Otsu, 1979) is used to determine the threshold for unwetted states (i.e. initial and loaded states), but for the collapsed state this method did not give satisfactory result. This is because clay-sized particles dispersed when the sample was saturated, and voxels that contains both sub-micron particles and voids would be blurred (partial-volume effect) and identified as solid. A consequence of this is that the two peaks (corresponding to solid and void) of the grayscale intensity histogram overlap considerably, in which case conventional thresholding 
methods generally do not apply. Thus, for the collapse state, the threshold value is determined by trial and error so that reasonable binary images were obtained. See Fig. 6(b) for a selected vertical slice of the binarized images.

The next step is to separate the solid phase in the binary result into individual particles, which is here referred to as 'segmentation'. However, accurate segmentation of particles is practically impossible for loess with wide range of complex structures and very small particles at the sub-micron level (see Fig. 6(a) and (b)). Problems such as oversegmentation (one particle separated into multiple objects) and under-segmentation (particles in contact but not separated) would be critical. To this end, the solid phase is 'eroded' by 4 voxels in order to facilitate the segmentation process. Erosion is a morphological operation in image processing, analogous to peeling a layer of skin with prescribed thickness, that shrinks large particles and removes very small particles. Segmentation is then carried out on the eroded solid phase (see Fig. 6(c)), resulting objects smaller than the original particles which will be hereafter referred to as 'particle nuclei'. The majority of particle nuclei preserve the morphological features of original particles reasonably well (see Fig. 6(d)). The dimensions of these original particles could be resestablished via 'dilation' (the inverse operation to 'erosion') using the same number of voxels. The analysis of kinematics of this research will be performed based on the particle nuclei. The tiny particles that have been suppressed by erosion are dismissed.

The eroded solid phase is segmented on the basis of a watershed algorithm (Beucher and Lantuejoul, 1979). In a typical watershed-based segmentation process, the region to be segmented is analogous to a number of connected basins. An initial water level is assigned to each basin and is allowed to head up until water levels in different basins meet to from watershed boundaries, separating different regions. In image processing, the depths of the abovementioned basins are usually obtained by calculating the 'distance map' from binary images. In a 3D distance map, typically a set of grayscale images, each voxel has a value equal to the shortest distance to the boundary of the region to be segmented. Here, the distance map is calculated from the eroded solid phase and serves as the benchmark for the following segmentation process.

Separating the particle nuclei still encounters considerable difficulty because of their large size range and irregular shapes, and segmentation could not be done effectively in a single step. To solve this problem, segmentation is carried out on different groups of nuclei, instead of on the whole eroded solid phase, through a sequence of iterative steps. The idea is similar to the adaptive watershed segmentation technique proposed by Kong and Fonseca (2018). In the current analysis, the eroded solid phase previously obtained is actually composed of many clusters made up by interconnected particle nuclei (see Fig. 7(a) for a simplified illustration of a such cluster). The maximum depth $h_{\max }$ (calculated from the distance map) within each cluster reflects approximately the size of the largest particle nucleus(nuclei) contained in the cluster. The first iteration, involving a series of watershed 
segmentation steps, is applied on the cluster(s) with the largest $h_{\max }$ (see Fig. 7(b-d) for an illustration of the process).

215 Beginning with the value of $h_{\max }-1$, the water level is progressively lowered by 1 voxel for each step of the watershed segmentation. In each step, former clusters are separated into multiple smaller clusters, among which only the ones with the same $h_{\max }$ is kept and are subject to the next segmentation step. The process is stopped when the remaining clusters could not be further segmented by lowering the water level (see Fig. 7(d)). By the end of each iteration, the remaining clusters are in fact well segmented particle nuclei, which are then taken out from the eroded solid phase. Subsequently, cluster(s) with the largest $h_{\max }$ are again selected from the rest of the solid phase for the next iteration. The effectiveness of this iterative technique diminishes as $h_{\max }$ becomes smaller, and clusters (containing very small particles) with $h_{\max }<3$ voxels are not considered in the analysis. Figure 8 shows the segmented particle nuclei within the sample in initial state, a total number of 5172 nuclei occupying $97.4 \%$ of the whole nuclei volume were obtained for the following analysis.

For a quantitative comparison with physically measured particle size distribution, the obtained particle nuclei are dilated by 4 voxels, recovering their original sizes before erosion. Figure 9 shows the particle size distribution measured by laser diffraction analysis and that obtained from image processing for the sample in initial state. The equivalent diameter (diameter of a sphere having the same volume as the particle) is used to measure the size of the segmented particles. Generally good agreement is observed for particle sizes larger than $30 \mu \mathrm{m}$, with minor discrepancies probably due to the inherent inaccuracy of the laser diffraction method in measuring particles with very irregular shapes (Abbireddy and Clayton, 2009; Blott and Pye, 2006). In contrast, for particles with smaller sizes, considerably more information is lost which is a side-effect of the erosion and segmentation processes. Nevertheless, the segmented particles include the majority of the skeleton particles and this allows to obtain representative results on their kinematics from the present analysis.

\section{Particle kinematics}

\subsection{Particle tracking}

Identification of the same set of particles within the samples in different states is crucial for the analysis of particle kinematics during the deformation process. This is achieved through a particle tracking technique named 'ID-track' proposed by Ando et al. (2012), which searches matching particles in different configurations according to their morphological properties. In this study, the equivalent diameter and sphericity of the segmented particle nuclei (referred to as 'particles' hereafter for brevity) are used as criteria for particle tracking, with a tolerance of $15 \%$. Each particle within the sample in the reference configuration (initial state) is assigned a unique ID and its counterparts meeting the criteria are searched within volumes in deformed configurations (loaded and collapsed states). In circumstances that more than one particle is found, the particle with the closest match is kept, while those 
particles with no matching counterparts are disregarded in further analysis. Assuming that the loess undergoes a globally (i.e. in the sample scale) uniform compression, the positions of particle centroids in deformed configurations can be estimated in the first instance based on global compressive strain, which is measured from the scanned images. This estimated position is then set to be the center of a cubic search window of size $30 \times 30 \times 30$ $\mu \mathrm{m}^{3}$ for the particle tracking process. Some 4175 and 3621 particles (from a total of 5172) are successfully correlated for loaded and flooded (collapsed) states respectively.

\subsection{Particle displacements}

Based on the sets of particles matched in different states through the ID-track method, particle displacements and rotations are determined. The displacements are calculated as the position changes of particle centroids in deformed configurations with respect to those in the initial configuration. Figs. 10(a) and (b) show the vertical displacement field of the particles relative to the base for loaded state and flooded (collapsed) state respectively. The magnitude of the displacement is indicated by the color range. The particle displacements at the top of the sample in collapsed state are about one order of magnitude larger than those in loaded state (see the range of the legend bars in Fig. 10), indicating significant collapse due to flooding of the sample. When the sample is not wetted, the displacement field is relatively uniform in the sense that particles at the same horizontal layer fall by approximately the same amount. In contrast, the displacement field of the flooded sample is much less homogeneous, suggesting that the loess microfabric is subject to varying levels of collapse at different locations within the sample.

Figures 11 (a) and (b) plot the components of particle displacements in different states against the initial coordinates along radial and axial directions respectively. Because of the thin disturbed layer at the sample-tube interface, the loess sample also undergoes lateral expansion as it is axially compressed. It can be seen that each set of displacement data can be regarded as values fluctuating about a straight fit line, the slope of which corresponds approximately to the global normal strain. The fluctuation is far more elevated for the flooded (collapsed) sample than for the unwetted sample, indicating a more heterogenous local displacement field. We express therefore the total particle displacements as the sum of global and local displacement components:

$$
\begin{aligned}
& u_{r}(x, y, z)=A_{r} r+B_{r}+u_{r}^{\prime}(x, y, z) \\
& u_{z}(x, y, z)=A_{z} z+B_{z}+u_{z}^{\prime}(x, y, z)
\end{aligned}
$$

where $u_{\alpha}(x, y, z)$ and $u_{\alpha}^{\prime}(x, y, z)(\alpha=r, z)$ denote respectively total and local particle displacements, and the first two terms on the right side of each expression represent the global displacement components that depend linearly on the initial particle coordinates. The coefficients $A_{\alpha}$ and $B_{\alpha}(\alpha=r, z)$ can be determined by linear fitting of the displacement data. Figure 12 illustrates the decomposition of particle displacements by plotting the displacement 
vectors on the identified particles within a selected vertical slice (in $Y-Z$ plane with the coordinate origin situated at the base center). Tomography images of the same vertical slice in initial and collapsed states are shown in Figs. 12(a) and (b) as a reference. In the total displacement vector field (Fig. 11(c)), it is largely impossible to distinguish local from global displacements. However, after the global components are filtered out (Fig. 12(f)), the pattern of local displacements of the loess micro-fabric can be seen clearly. This issue will be addressed in the next section.

In order to quantify the local variation of the particle displacements along particular directions, we define the coefficients of heterogeneity along radial and axial directions, $C_{H r}$ and $C_{H z}$, as the average of local displacement magnitudes normalized by the average grain size:

$$
C_{H \alpha}=\frac{\frac{1}{N}\left(\sum_{i=1}^{N}\left|u_{\alpha(i)}^{\prime}\right|\right)}{D_{50}},(\alpha=r, z)
$$

where $u_{\alpha(i)}^{\prime}(\alpha=r, z)$ is the local displacement component of the $i$-th particle along $\alpha$ (radial or axial) direction and $N$ the total number of the particles. Table 1 lists the coefficients of heterogeneity along different directions for sample in loaded and flooded (collapsed) states. The results show typically larger coefficients of heterogeneity for the collapsed sample compared to those for loaded but unwetted sample, suggesting that the level of deformation heterogeneity increases with the amount of global deformation. These coefficients of heterogeneity are potentially powerful expressions enabling quantitative comparisons between tests on different samples.

\subsection{Particle rotations}

To measure particle rotations, the orientation of a 3D vector is here described in a horizontal coordinate system with the azimuthal and altitude angles, $\theta$ and $\varphi$, as independent coordinates (Fig. 13(a)). The orientation of each particle is determined by the three orthogonal eigenvectors which can be calculated from the tensor of moment of inertia. The two eigenvectors corresponding to the largest and smallest eigenvalues are provided in Avizo, and the third one is obtained by right hand rule due to the orthogonality of eigenvectors. Because of the finite range of altitude angles $\left(0 \sim 90^{\circ}\right)$, the direction of eigenvectors might 'flip'. For example, a rotation by 2 degrees in the vertical plane from $(\theta, \varphi)=\left(45^{\circ}, 89^{\circ}\right)$ will result in $\left(-135^{\circ}, 89^{\circ}\right)$. This is corrected by limiting the absolute rotation angles of eigenvectors within the range between 0 and $90^{\circ}$, which is reasonable considering the small level of particle movements in this study. The orientation changes of eigenvectors from initial to the deformed configuration derive the rotation matrix, from which a rotation angle, $\psi$, around an unique rotation axis are calculated using Euler's rotation theorem and are used to quantify the particle rotations during the deformation process, see Fig. 13(a).

Figure 13(b) plots the field of particle rotation magnitude in collapsed state, showing a haphazard distribution within the sample. Figure 13(c) shows the number fractions of particles that undergo different amount of rotations. 
We see that the fraction decreases for larger rotation magnitudes, with a vast majority of particles rotating by an angle smaller than $30^{\circ}$. The number fractions of particles that rotate with respect to differently orientated axis are shown in Fig. 13(d) in terms of the altitude angle, $\varphi$, and the acute angle formed by the rotation axis and $X$ axis, min $\left(|\theta|, 180^{\circ}-|\theta|\right)$, corresponding respectively to vertical and horizontal orientations both ranging from 0 to $90^{\circ}$. An increasing number of particles can be seen that rotate about axis having larger altitude angles, which implies that the particles tend to rotate vertically rather than horizontally, i.e., around axis perpendicular to the direction of major principal stress. It is worth mentioning that this trend is found to be true irrespective of the initial orientation or morphology of the particles. The approximately constant fraction of particles rotating about axis having different horizontal orientations indicates that the rotation axis has no preferred azimuthal direction, as expected.

Figure 14(a) plots the rotation angles of individual particles against their equivalent diameters in order to assess the dependence of particle size on rotation magnitude. For relatively small particles, for example those with equivalent diameter of less than $30 \mu \mathrm{m}$, the rotation magnitudes cover a wide range from about 0 to $100^{\circ}$, although a concentration towards smaller angles can be clearly seen. In contrast, as the equivalent diameter increases, the rotation angles converge gradually to a very low level, which might be explained as follows. Since the particles in loess are generally far from spherical in shape, it thus requires an adequate void space for a finite particle rotation to take place. From a statistical point of view, a large particle is more likely to be 'embedded' in smaller particles or fine particle aggregates that provide practically no voids having sizes comparable to that of the large particle. However, smaller particles are more likely to be surrounded by particles of similar sizes and are therefore less constrained for a rotation. Figure 14 (b) shows the dependency of rotation angles on particle flatness, which is defined as the ratio of the smallest to the intermediate eigenvalue of the tensor of moment of inertia (smaller flatness for more platy particles) in order to study the influence of particle morphology. As opposed to the case for particle size, the particle morphology seems to have a minor effect on the level of rotation as shown by the result. It is worth mentioning that rotation of particles in a multi-modal granular medium is a complex issue depending on other factors besides particle size and morphology, which are not the primary focus of this study.

\section{Strain analysis}

The identified and tracked particles are limited in number and vary in size and shape. As a consequence, the strain field could not be properly obtained using common homogenization methods based on the particle kinematics. We therefore develops an approach to calculate the volumetric strain as explained in the following. First, the local porosity $n$ at an arbitrary point is calculated from the binary images within a cubic box, whose edge length $L$ represents the scale of interest for the strain calculation. By fitting the displacements of tracked particles in deformed states, any point within the sample could be roughly mapped between different configurations (see Fig. 15) . This allows the change of local porosity adjacent to this arbitrary point to be evaluated. The local volumetric strain (with 
compressive stains taken to be positive) is calculated by:

$$
\varepsilon_{v}=\frac{n-n_{0}}{1-n}
$$

where $n_{0}$ and $n$ denote respectively the local porosities in initial and deformed configurations. To quantitatively assess the relationship between deformation characteristics and loess microfabric, the local volumetric strains are plotted against initial local porosities at different scales (see Fig. 16). It can be seen that a clear positive dependence exists between the local strain and local porosity irrespective of the scale considered, indicating that relatively loose regions tend to undergo a larger amount of contraction during the collapse. Moreover, as the scale enlarges, the variation range of the local strain as well as that of the local porosity values converge, showing an upscale averaging effect. For $L=50 \mu \mathrm{m}$ (Fig. 16(a)), the size of the cubic box is about the same as the scale of a (large) particle/pore, we see some quasi-zero porosity values which correspond to cases where the box is entirely situated within a large pore. The level of deformation evaluated at this scale is also more drastic compared to that at larger scales (Fig. 17(b) and (c)). It could be reasonably expected that when the scale of interest increases further, the local strain and porosity would progressively converge to unique values, which approximately equal to the porosity measured at macroscale and global strains (for example those associated with the commonly used coefficient of collapsibility, $\delta$; China National Standards (CNS) GB50025-2004 (Standardization Administration of China (SAC), Ministry of Construction, 2004).

Selected slices extracted from the 3D images are used to visualize the spatial distribution of volumetric strain and to make comparison with former results of the particle kinematics, see details in Figs. 17 and 18 for slices in vertical and horizontal plane respectively. Figure 17(a) and (b) present respectively the grayscale slices in the same vertical plane ( $Y-Z$ plane) in initial and collapsed states. It can be observed clearly that the microfabric has evolved to a denser state and the (relatively) large pore at upper-right of the slice is notably contracted. In Fig. 17(c) the local displacement vectors are plotted upon identified and tracked particles within the same slice. The vectors that are adjacent to the above-mentioned pore lean towards the void, indicating contraction of the pore. Figure 17(d) shows the local porosity contours at different scales. It can be seen that the porosity calculated using the current method is able to reflect the microfabric characteristics shown in Fig. 17(a), with different spatial resolutions. The local volumetric strain fields are shown in Fig. 17(e), which show very similar pattern as those of the local porosity distribution despite of different scales of interest, demonstrating the strong interdependency between the local deformation and microfabric as concluded from Fig. 16. The same conclusion could be drawn from an analysis in a horizontal plane ( $X$ - $Y$ plane), see Fig. 18.

\section{Inter-particle contact}


The evolution of inter-particle contacts (i.e. coordination number, branch vector, etc.) is a fundamental aspect

for characterizing the change in microfabric. Dijkstra et al. (1995) postulated a theoretical model describing the process of hydro-consolidation in the context of particle packing transformation. They suggested that the waterinduced collapse of loess is accompanied by change of angular separation and breaking of bridging structures between particles that are assumed to be platy (see Fig. 7 in Dijkstra et al., 1995), and this still requires experimental evidence for validation. The state-of-the-art employed in this work has disadvantages in the sense that it is impossible to extract and identify all the particles within the sample (i.e. most identified particles are separated from one another), therefore realistic coordination numbers or the full set of branch vectors could not be obtained. However, the current technique does provide the possibility to observe the evolving contact features within some 'clusters' that are composed of inter-connected particles. It has been possible to find several such clusters comprising more than a hundred connected particles from the whole set of identified particles and to analyze their contact characteristics. The same group of particles that compose the undeformed cluster are identified in collapsed state, where some of the particles were no longer inter-connected. The contact occurrence within the cluster in both configurations is then detected and the branch vectors are obtained using the built-in PNM module in Avizo, which is originally designated to analyze pore connectivity.

Table 2 gives the change of contact number during collapse as well as the mean altitude angles of branch vectors in initial and collapsed states for two clusters containing 182 and 135 individual particles, which are hereafter referred to as clusters A and B. The statistics show that during collapse, more than half of the original inter-particle contacts are broken. At the same time a few new contacts are formed, indicating that some former separated particles are brought into contact during the sample deformation. The contact number change implies an apparently decreasing mean coordination number as the loess densifies. However, this is an artifact of the limited number of identified particles. In real collapse process, many more contacts will be formed between particles within the cluster and nearby particles which are not identified here. It can be reasonably expected that this will lead to an increase in mean coordination number, but the micro-fabric transformations observed here are clearly much more complex than initially suggested in Dijkstra et al. (1995). What this research visualizes and quantifies, for the first time, are the complexities involved in kinematics of individual particles during the collapse process. An increase of mean altitude angle of the branch vectors by approximately 3 degrees is found for both clusters, suggesting that the microfabric is contracted along the vertical direction (direction of major principle stress), as expected.

For a visual inspection of the contact evolution, Figs. 19 and 20 show the constituting particles as well as the branch vector diagrams in different states for clusters A and B respectively. A comprehensive re-arrangement of particles caused by the collapse process is clearly evidenced by the broken and newly formed contacts (branch 
vectors). Although the contact relationship between particles is significantly altered, the relative position of particles does not change as dramatically as anticipated, and many 'bridging' structures formed by particles still persist even upon flooding (see for example the results for cluster B in Fig. 19). This does not appear to support the particle level collapse mechanism proposed in Dijkstra et al. (1995). However, further experimental investigation at the microscale is required to reveal what truly happens in the collapse (hydro-consolidation) process. This should include further improvement in the detail of the imaging and segmentation techniques in order to yield more realistic coordination numbers that allowing direct comparison with the theoretical postulation.

\section{Conclusions}

The particle kinematics and microfabric evolution within the same loess sample during the hydro-consolidation process have been characterized for the first time (to the best of our knowledge). The microfabric features of the sample in initial, loaded and flooded (i.e. collapsed) states have been captured with the help of an experimental apparatus designed especially for collapse test of several millimeters large specimens combined with the X-ray tomography technique with one-micron spatial resolution. An iterative segmentation technique in combination with particle tracking (the 'ID-track' method) have been employed to identify and track individual particles within the sample in different configurations, allowing the particle displacement and rotation to be determined. The displacement field in the collapsed configuration exhibit a higher level of heterogeneity compared to that within the loaded (but unwetted) sample. By defining a coefficient of heterogeneity $\left(C_{H}\right)$, the local heterogeneity in particle displacement field has been quantified. This is potentially a powerful expression enabling quantitative comparison between tests on different samples. It is shown that the deformation of the flooded (collapsed) sample exhibit significant heterogeneity compared to the loaded but unwetted sample. In addition, the particles tend to rotate vertically rather than horizontally during collapse, with the rotation magnitudes controlled predominantly by particle size rather than being influenced by the particle shape. The local porosity and local volumetric strain field show very similar patterns at different scales, which implies that the level of local deformation is highly dependent on the local density of the microfabric, similar to the loess porosity-collapsibility relationship established at the macroscale. Clusters comprising inter-connected particles have been analysed to study the contact evolution during the collapse process. A large number of contacts are found to be broken while a few new contacts between the originally separated particles are formed. It is important to note that there will be many more contacts formed in the denser, collapsed fabric, but these involve new relationships of the originally selected particles with other particles outside of this selection. This supports the observation that the collapse process involves substantial re-arrangement of particles and the formation of a whole new set of inter-particle connections that is much more complex than previously assumed (see e.g. Dijkstra et al., 1995). 
The re-arrangement of particles during hydro-consolidation is clearly demonstrated, suggesting that the state-

of-the-art used in this work could serve as an effective tool that might contribute to further progress in the particle packing theory for loess. The visualization and enhanced capability for quantification of individual particle dynamics during the collapse process provided by this research offers exciting new avenues for the interpretation of other excellent loess collapse studies, such as those by Shao et al. (2018) and Wen and Yan (2014). Although an incremental step has been made towards a better understanding of the loess collapse mechanism, the approaches employed in this work have some limitations. While the particle kinematics and strain fields have been obtained based on sample configurations before and after collapse, the question of how loess microfabric evolves temporally during the hydro-consolidation process is beyond the capability of the methods used in this research. The uncontrollable and extremely fast progress of water-induced collapse poses a huge challenge to the experimentation and imaging technique. Numerical tools such as the discrete element method provide a potential opportunity to address this issue (Cundall and Strack, 1979, Jiang et al., 2014) and the micron-scale observational capability from this research could serve as a reference for the validation of simulated results (see e.g. Jiang et al., 2017; Jiang et al., 2020). Another limitation of the present study lies in that only skeleton particles of large and intermediate sizes have been identified whereas very small silts and clay-sized particles that occupy also an important volume fraction were practically neglected, limited by the spatial resolution of X-ray imaging as well as the effectiveness of image processing. This also hindered a more comprehensive investigation of the contact evolution. Therefore, it is suggested to proceed in further research where numerical studies as well as physio-chemical methods could be involved in order to fully assess the loess collapse phenomenon, which is intrinsically a multi-scale and multidisciplinary problem.

\section{Acknowledgements}

This research was carried out thanks to the support of National Natural Science of China (grand numbers: 41902281 and 41630634). The author also would like to acknowledge Dr. Cao Chunjie (Carl Zeiss (Shanghai) Co. Ltd.) and Dr. Zhou Runqing (Core Labs of IGGCAS) for their valuable technical support on this work.

\section{References}

Abbireddy, C.O.R., Clayton, C.R.I., 2009. A review of modern particle sizing methods. Proceedings of the Institution of Civil Engineers - Geotechnical Engineering 162 (4), 193-201. https://doi.org/10.1680/geng.2009.162.4.193

Ando, E., Hall, S.A., Viggiani, G., Desrues, J., Besuelle, P., 2012. Grain-scale experimental investigation of localised deformation in sand: a discrete particle tracking approach. Acta. Geotech. 7 (1), 1-13. https://doi.org/10.1007/s11440-011-0151-6

Assadi-Langroudi, A., Ng'ambi, S., Smalley, I.J., 2018. Loess as a collapsible soil: Some basic particle packing aspects. 
Barden, L., Mcgown, A., Collins, K., 1973. The collapse mechanism in partly saturated soil. Eng. Geol. 7 (1), $49-60$. https://doi.org/10.1016/0013-7952(73)90006-9

Beucher, S., Lantuejoul, C. 1979. Use of watersheds in contour detection. Proceedings of international workshop on image processing: real-time edge and motion detection/estimation, 1979 Rennes, France. 17-21.

Blott, S.J., Pye, K., 2006. Particle size distribution analysis of sand-sized particles by laser diffraction: an experimental investigation of instrument sensitivity and the effects of particle shape. Sedimentology 53 (3), 671-685. https://doi.org/10.1111/j.1365-3091.2006.00786.x

Cheng, Z., Wang, J.F., 2018. Experimental investigation of inter-particle contact evolution of sheared granular materials using X-ray micro-tomography. Soils Found. 58 (6), 1492-1510. https://doi.org/10.1016/j.sandf.2018.08.008

Cnudde, V., Boone, M.N., 2013. High-resolution X-ray computed tomography in geosciences: A review of the current technology and applications. Earth-Sci. Rev. 123, 1-17. https://doi.org/10.1016/j.earscirev.2013.04.003

Cui, Y.J., Terpereau, J.M., Marcial, D., Delage, P., Antoine, P., Marchadier, G., Ye, W.M. 2004. A geological and geotechnical characterisation of the loess of Northern France. Advances in geotechnical engineering: The Skempton conference. https://doi.org/10.1680/aigev1.32644.0019

Cundall, P.A., Strack, O.D.L., 1979. A discrete numerical model for granular assemblies. Geotechnique 29 (1), 47-65. https://doi.org/10.1680/geot.1979.29.1.47

Derbyshire, E., Mellors, T.W., 1988. Geological and geotechnical characteristics of some loess and loessic soils from China and Britain: A comparison. Eng. Geol. 25 (2), 135-175. https://doi.org/10.1016/0013-7952(88)90024-5

Dijkstra, T.A., Smalley, I.J., Rogers, C.D.F., 1995. Particle packing in loess deposits and the problem of structure collapse and hydroconsolidation. Eng. Geol. 40 (1), 49-64. https://doi.org/10.1016/0013-7952(95)00022-4

Fonseca, J., O'sullivan, C., Coop, M.R., Lee, P.D., 2013a. Quantifying the evolution of soil fabric during shearing using directional parameters. Geotechnique 63 (6), 487-499. https://doi.org/10.1680/geot.12.P.003

Fonseca, J., O'sullivan, C., Coop, M.R., Lee, P.D., 2013b. Quantifying the evolution of soil fabric during shearing using scalar parameters. Geotechnique 63 (10), 818-829. https://doi.org/10.1680/geot.11.P.150

Gao, G.R., 1981. Classification of microstructures of loess in China and their collapsibility. Sci. Sin. 24 (7), $962-970$.

Hall, S.A., Bornert, M., Desrues, J., Pannier, Y., Lenoir, N., Viggiani, G., Besuelle, P., 2010. Discrete and continuum analysis of localised deformation in sand using X-ray mu CT and volumetric digital image correlation. Geotechnique 60 (5), 315-322. https://doi.org/10.1680/geot.2010.60.5.315

Jiang, M.J., Li, T., Thornton, C., Hu, H.J., 2017. Wetting-Induced Collapse Behavior of Unsaturated and Structural Loess under Biaxial Tests Using Distinct Element Method. Int. J. Geomech. $17 \quad(1)$. https://doi.org/10.1061/(ASCE)GM.1943-5622.0000693 
Jiang, M.J., Wang, Y., Lei, H., Li, T., Chen, Y. and Sun, R., 2020. DEM analyses of true triaxial and wetting tests on unsaturated structural loess. Japanese Geotechnical Society Special Publication, 8(6), 231-234.

Jiang, M.J., Zhang, F.G., Hu, H.J., Cui, Y.J., Peng, J.B., 2014. Structural characterization of natural loess and remolded loess under triaxial tests. Eng. Geol. 181, 249-260. https://doi.org/10.1016/j.enggeo.2014.07.021

Juang, C.H., Dijkstra, T.A., Wasowski, J., Meng, X.M., 2019. Loess geohazards research in China: Advances and challenges for mega engineering projects. Eng. Geol. 251, 1-10. https://doi.org/10.1016/j.enggeo.2019.01.019

Kong, D., Fonseca, J., 2018. Quantification of the morphology of shelly carbonate sands using 3D images. Geotechnique 68 (3), 249-261. https://doi.org/10.1680/jgeot.16.P.278

Kong, D., Fonseca, J., 2019. On the kinematics of shelly carbonate sand using X-ray micro tomography. Eng. Geol. 261, 105268. https://doi.org/10.1016/j.enggeo.2019.105268

Lei, X.Y., 1989. The relationship between microtexture types and indices of physico-mechanical properties of loess in China. Acta Geol. Sin. 2 (4), 433-443. https://doi.org/10.1111/j.1755-6724.1989.mp2004008.x

Li, X.A., Li, L.C., 2017. Quantification of the pore structures of Malan loess and the effects on loess permeability and environmental significance, Shaanxi Province, China: an experimental study. Environ. Earth Sci. 76 (15), 523. https://doi.org/10.1007/s12665-017-6855-7

Liu, Z., Liu, F., Ma, F., Wang, M., Bai, X., Zheng, Y., Yin, H., Zhang, G., 2015. Collapsibility, composition, and microstructure of loess in China. Can. Geotech. J. 53 (4), 673-686. https://doi.org/10.1139/cgj-2015-0285

Luo, H., Wu, F., Chang, J., Xu, J., 2018. Microstructural constraints on geotechnical properties of Malan Loess: A case study from Zhaojiaan landslide in Shaanxi province, China. Eng. Geol. 236, 60-69. https://doi.org/10.1016/j.enggeo.2017.11.002

Ng, C.W.W., Mu, Q.Y., Zhou, C., 2016. Effects of soil structure on the shear behaviour of an unsaturated loess at different suctions and temperatures. Can. Geotech. J. 54 (2), 270-279. https://doi.org/10.1139/cgj-2016-0272

Otsu, N., 1979. A Threshold Selection Method from Gray-Level Histograms. IEEE Transactions on Systems, Man, and Cybernetics 9 (1), 62-66. https://doi.org/10.1109/TSMC.1979.4310076

Peng, J.B., Qi, S.W., Williams, A., Dijkstra, T.A., 2018. Preface to the special issue on "Loess engineering properties and loess geohazards”. Eng. Geol. 236, 1-3. https://doi.org/10.1016/j.enggeo.2017.11.017

Rogers, C.D.F., Dijkstra, T.A., Smalley, I.J., 1994. Hydroconsolidation and subsidence of loess: Studies from China, Russia, North America and Europe: In memory of Jan Sajgalik. Eng. Geol. 37 (2), 83-113. https://doi.org/10.1016/0013-7952(94)90045-0

Shao, X., Zhang, H., Tan, Y., 2018. Collapse behavior and microstructural alteration of remolded loess under graded wetting tests. Eng. Geol. 233, 11-22. https://doi.org/10.1016/j.enggeo.2017.11.025

Standardization Administration of China (SAC), Ministry of Construction, 2004. China National Standards GB50025- 

(In Chinese).

Viggiani, G., Tengattini, A. 2019. Recent developments in laboratory testing of geomaterials with emphasis on imaging. Proceedings of the XVII European Conference on Soil Mechanics and Geotechnical Engineering. Reykjavik, Iceland.

Wang, J.D., Li, P., Ma, Y., Vanapalli, S.K., Wang, X.G., 2019. Change in pore-size distribution of collapsible loess due to loading and inundating. Acta. Geotech. 15, 1081-1094. https://doi.org/10.1007/s11440-019-00815-9

Wang, L.Q., Shao, S.J., She, F.T., 2020. A new method for evaluating loess collapsibility and its application. Eng. Geol. 264, 105376. https://doi.org/10.1016/j.enggeo.2019.105376

Watanabe, Y., Lenoir, N., Otani, J., Nakai, T., 2012. Displacement in sand under triaxial compression by tracking soil particles on X-ray CT data. Soils Found. 52 (2), 312-320. https://doi.org/10.1016/j.sandf.2012.02.008

Wei, T.T., Fan, W., Yuan, W.N., Wei, Y.N., Yu, B., 2019. Three-dimensional pore network characterization of loess and paleosol stratigraphy from South Jingyang Plateau, China. Environ. Earth Sci. 78 (11), 333. https://doi.org/10.1007/s12665-019-8331-z

Wei, Y.N., Fan, W., Yu, B., Deng, L.S., Wei, T.T., 2020. Characterization and evolution of three-dimensional microstructure of Malan loess. Catena 192, 104585. https://doi.org/10.1016/j.catena.2020.104585

Wen, B.P., Yan, Y.J., 2014. Influence of structure on shear characteristics of the unsaturated loess in Lanzhou, China. Eng. Geol. 168, 46-58. https://doi.org/10.1016/j.enggeo.2013.10.023

Xu, L., Coop, M.R., 2016. Influence of structure on the behavior of a saturated clayey loess. Can. Geotech. J. 53 (6), 1026-1037. https://doi.org/10.1139/cgj-2015-0200

Yang, Y.L., 1988. Study on collapsible mechanism of loess soils. Sci. China Ser. B 7, 756-766 (in Chinese). 
Tables

Table 1. Coefficients of heterogeneity of the loess sample in different states

\begin{tabular}{c|c|c}
\hline Sample state & $C_{H r}$ & $C_{H z}$ \\
\hline Loaded & 0.031 & 0.023 \\
\hline Flooded (collapsed) & 0.150 & 0.146 \\
\hline
\end{tabular}

Table 2. Contact evolution of clusters of initially interconnected particles

\begin{tabular}{c|c|c|c|c|c|c}
\hline \multirow{2}{*}{ Cluster ID } & \multirow{2}{*}{$\begin{array}{c}\text { Number of } \\
\text { particles }\end{array}$} & \multicolumn{3}{|c|}{ Number of contacts } & \multicolumn{2}{c}{ Mean branch vector orientation $\left({ }^{\circ}\right)$} \\
\cline { 3 - 7 } & 182 & Initial & Broken & Newly formed & Initial & Collapsed \\
\hline A & 135 & 146 & 108 & 6 & 60.0 & 62.9 \\
\hline B & 137 & 87 & 7 & 58.1 & 62.2 \\
\hline
\end{tabular}


Figures

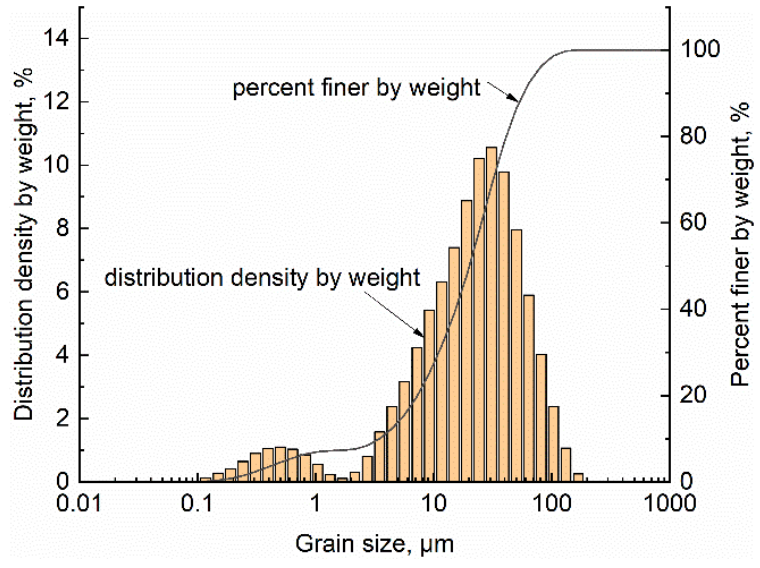

(a)

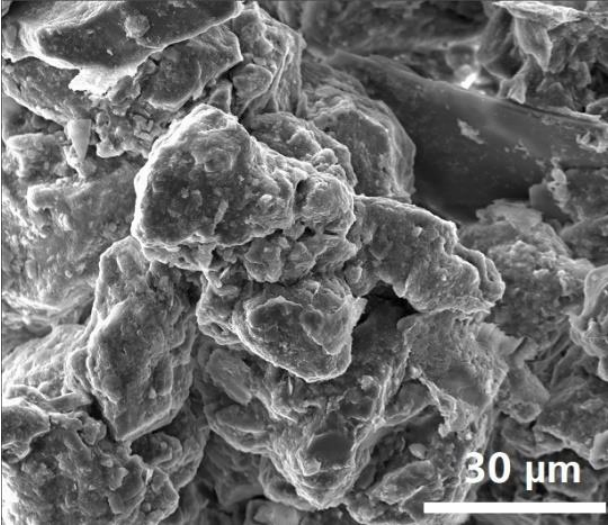

(b)

Fig. 1. (a) Particle size distribution of the loess studied (with 0.1 as a log bin step for the distribution density); (b) SEM photograph showing microfabric details

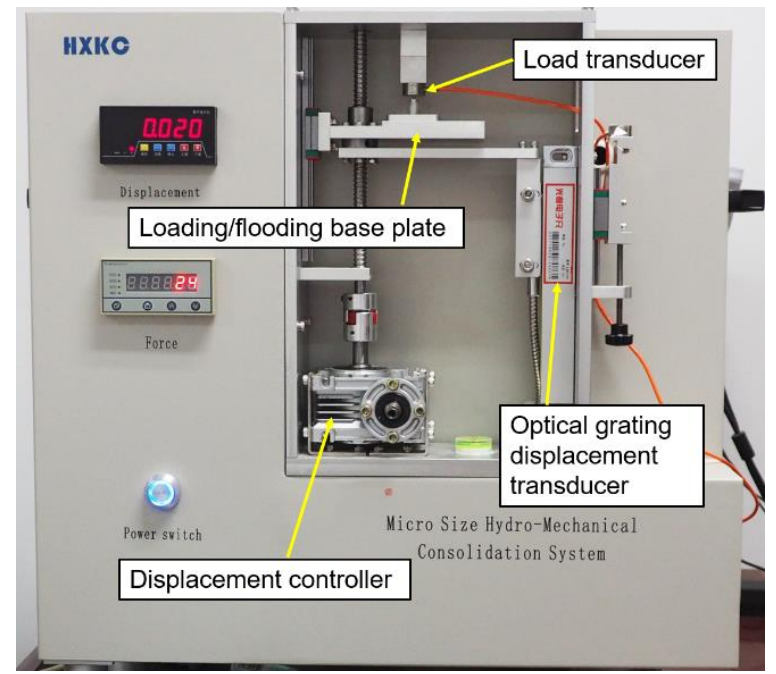

(a)

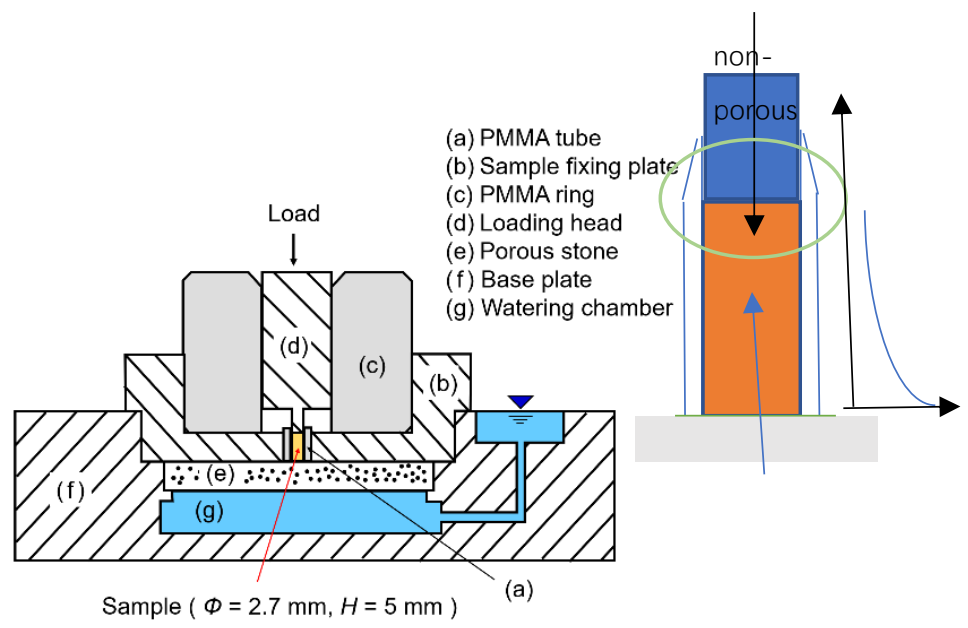

(b)

Fig. 2. (a) Experimental apparatus designed for collapse test on mini-sized samples; (b) schematic of the key components of the loading/flooding base plate 


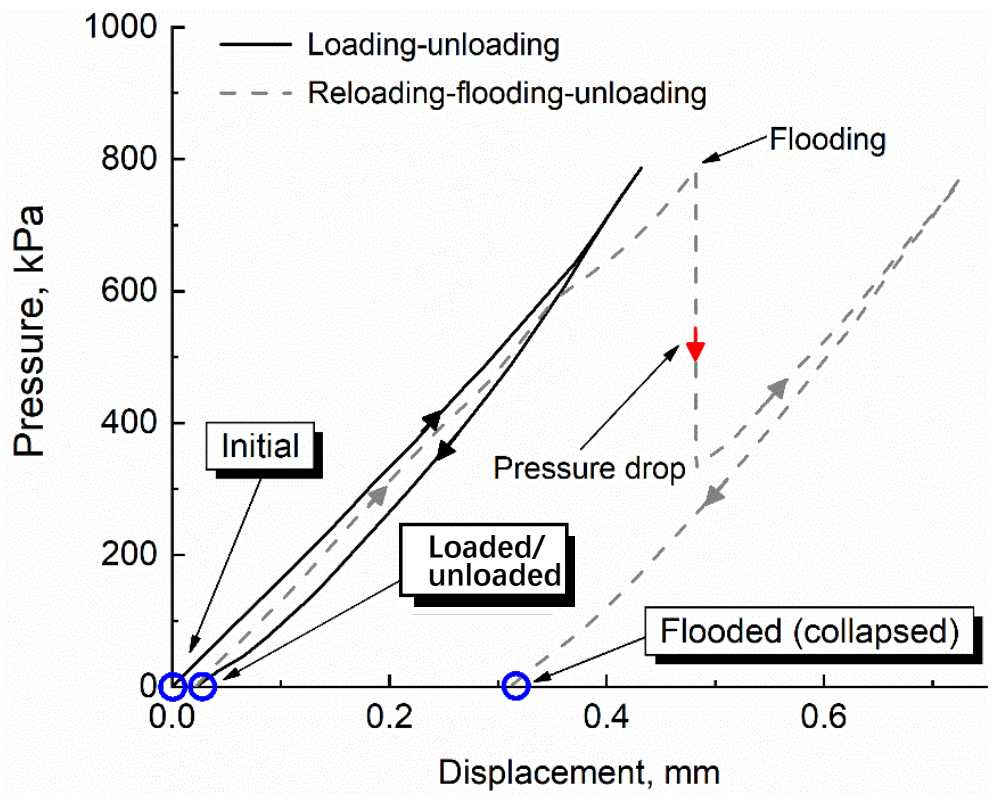

Fig. 3. Loess sample response curves in terms of measured pressure against axial displacement during the test, marked with key response features and the prescribed states to be subject to $\mu$-CT scan 


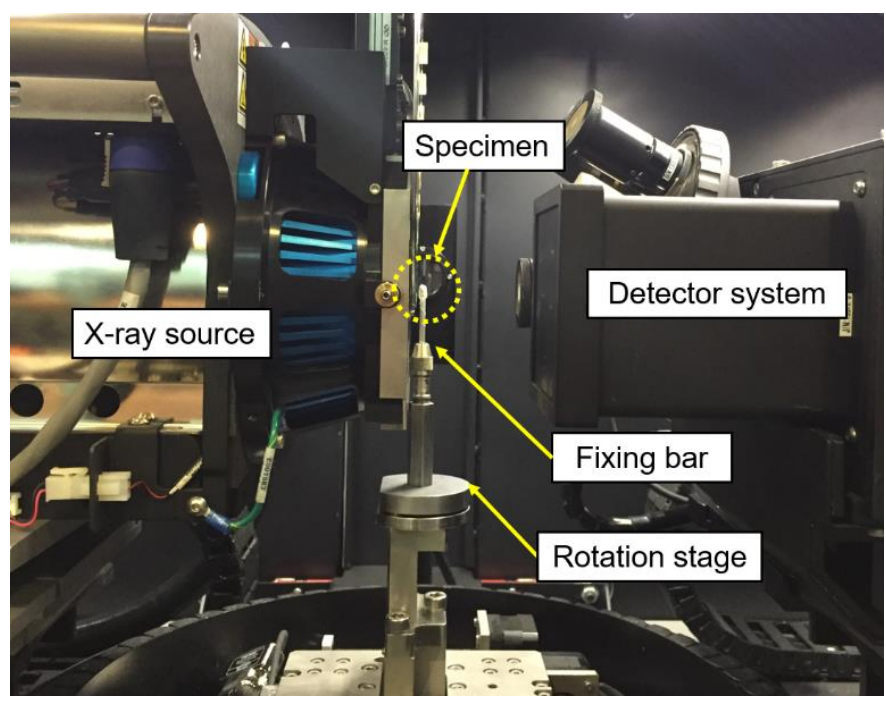

(a)
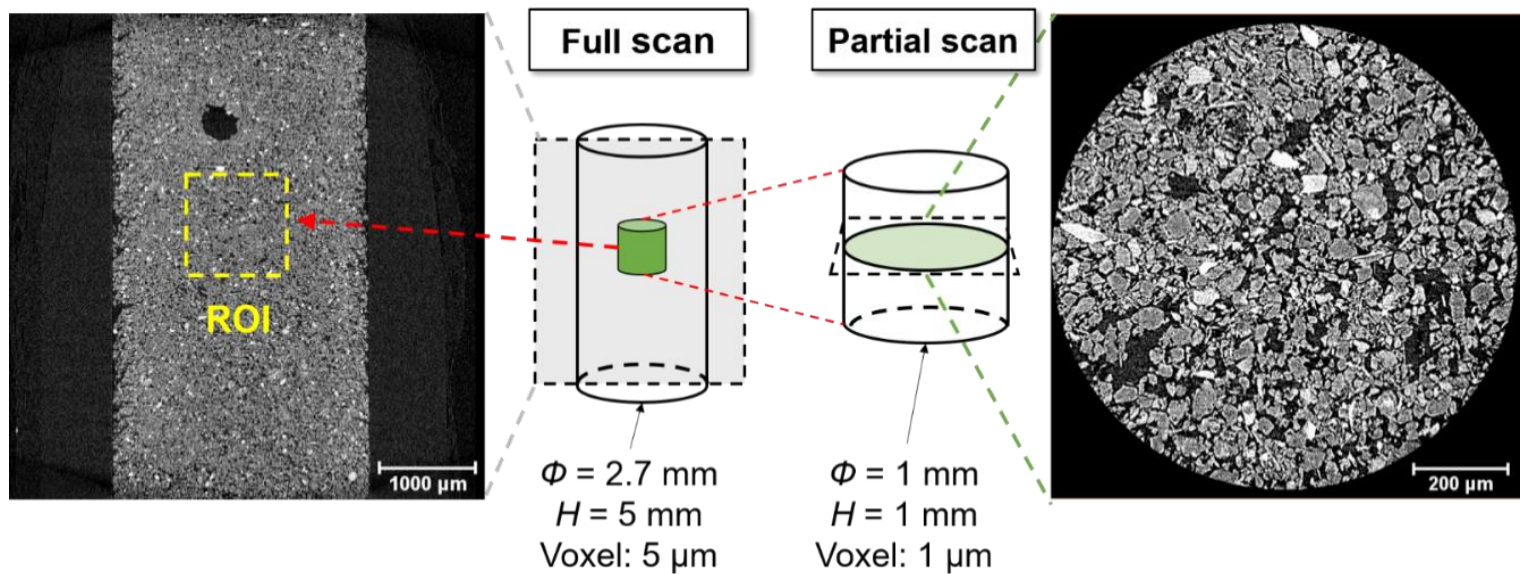

(b)

Fig. 4. (a) Details of the X-ray tomography set-up; (b) schematic of full and partial scans involving the output image slices 


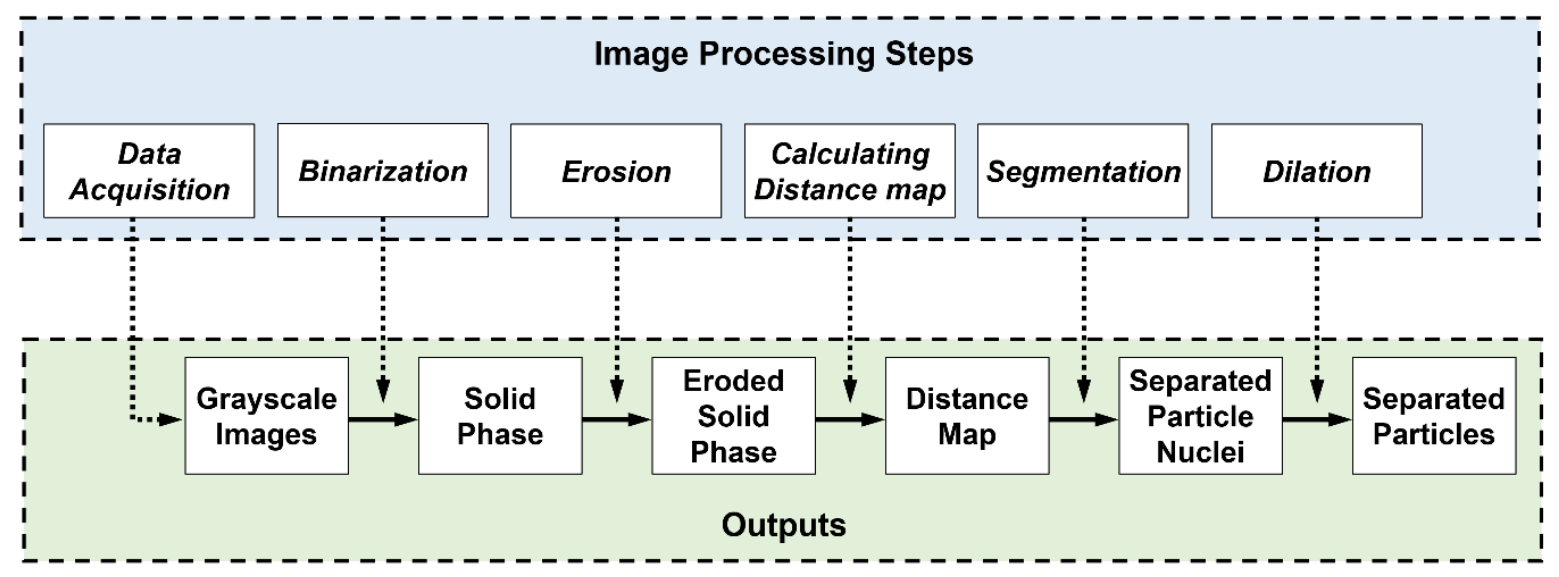

Fig. 5. Flowchart of the image processing steps and outputs

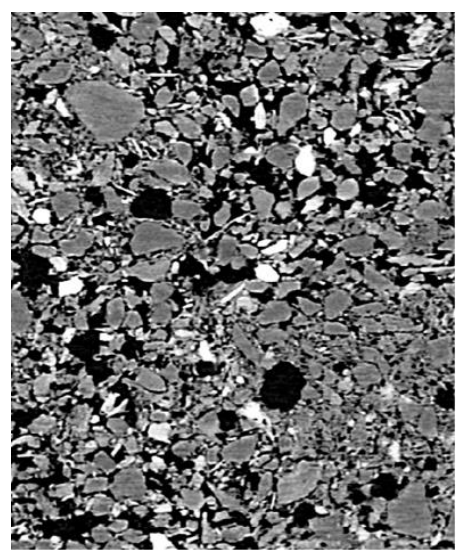

(a)

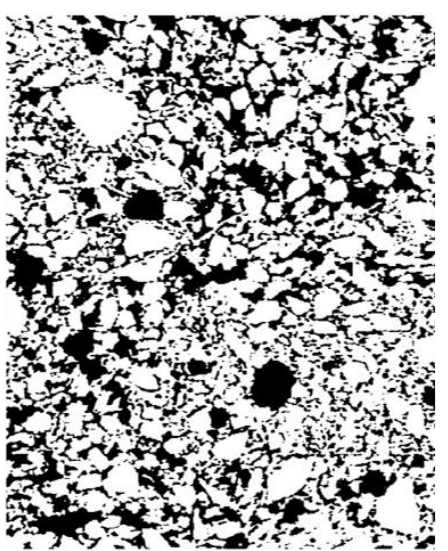

(b)

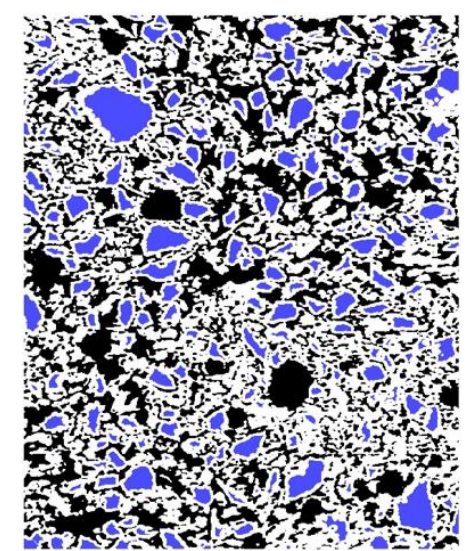

(c)

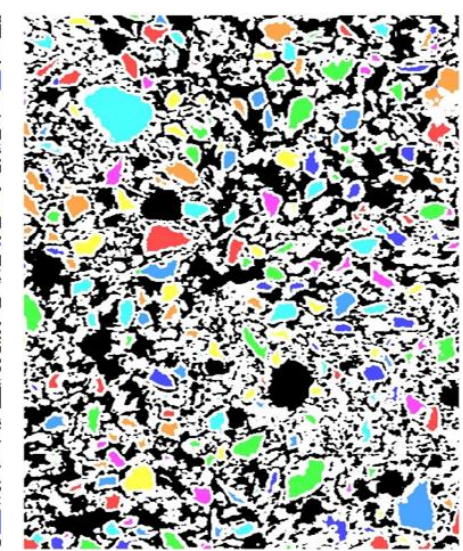

(d)

Fig. 6. Vertical slices showing results from main steps of image processing: (a) grayscale image; (b) binarized image; (c) unseparated particle nuclei; (d) separated particle nuclei (labelled with different colors). The slices are extracted from the sample volume in initial state and have dimension of $695 \times 767 \mu \mathrm{m}^{2}$ 


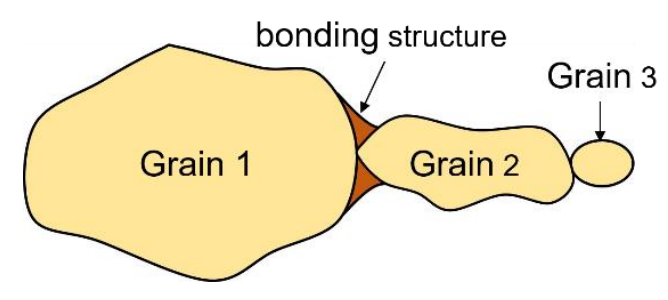

(a)

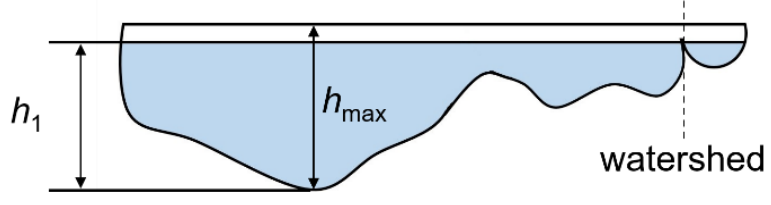

(b)

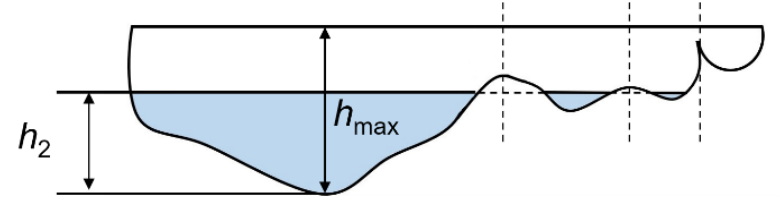

(c)

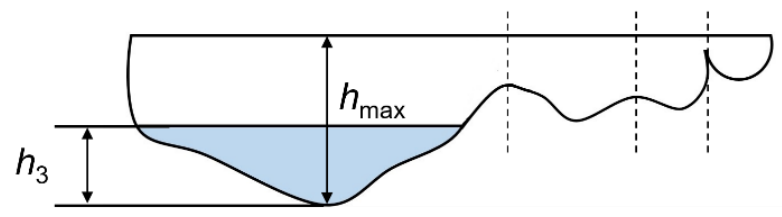

(d)

Fig. 7. (a) Illustration of a cluster comprising connected particles; (b) to (c) progressively refined watershed segmentation of the cluster 


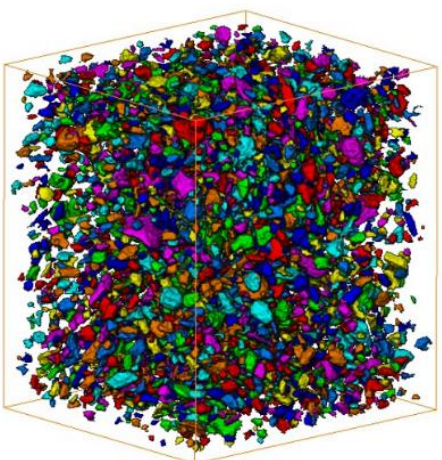

$h_{\text {max }}>=3$ voxels

No. of nuclei: 5172

Volume percentage: $97.4 \%$

(a)

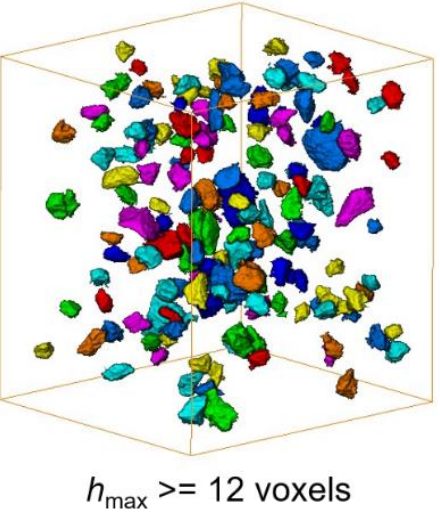

No. of nuclei: 172

Volume percentage: $65.9 \%$

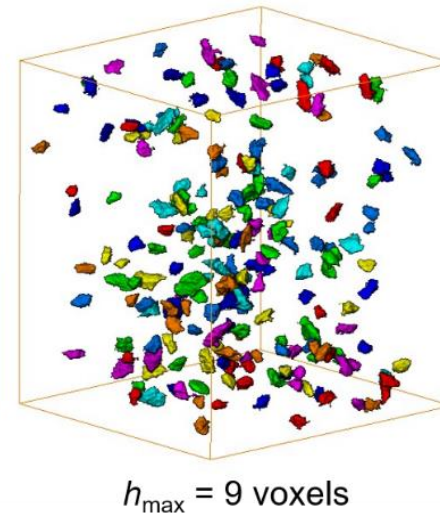

No. of nuclei: 211

Volume percentage: $6.8 \%$

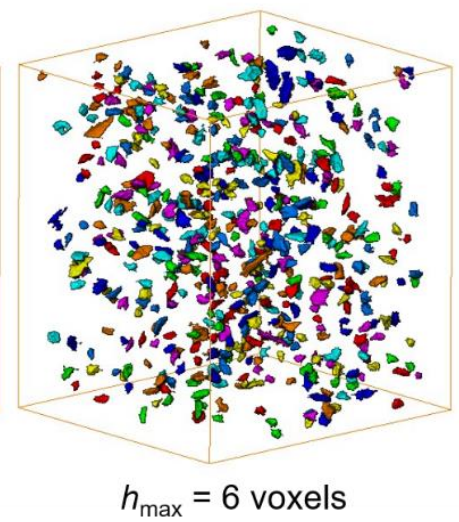

No. of nuclei: 580

Volume percentage: $6.4 \%$

(b)

(c)

(d)

Fig. 8. Result of the segmentation process: (a) all particle nuclei separated from the sample in initial state; (b) to (d) particle nuclei with different $h_{\max }$.

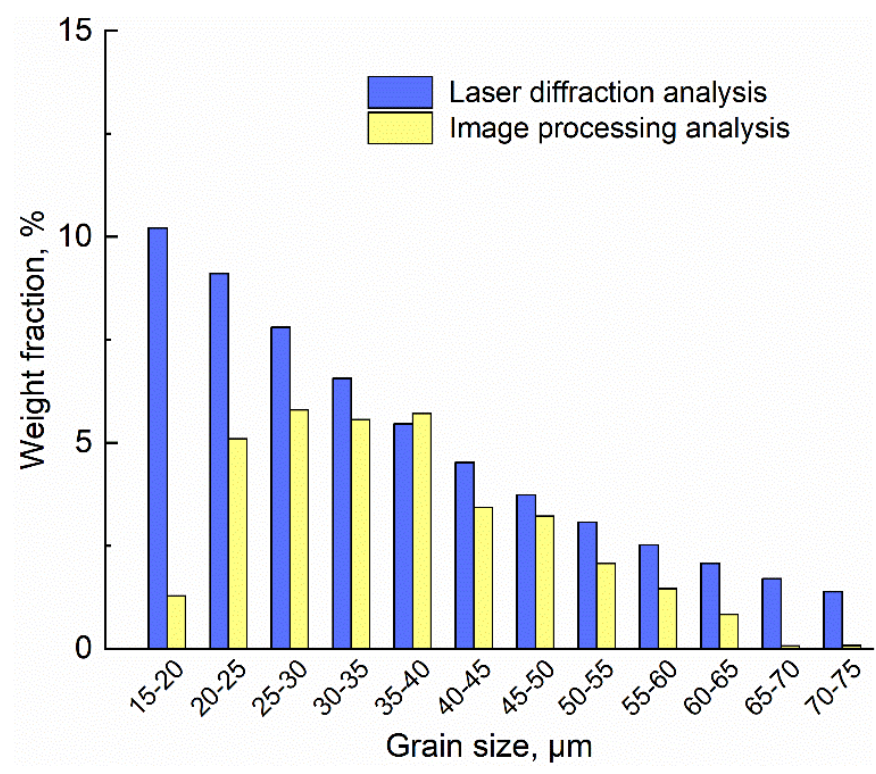

Fig. 9. Comparison between the particle size distribution measured by laser diffraction and that obtained from current image processing analysis (results from the sample in initial state) 


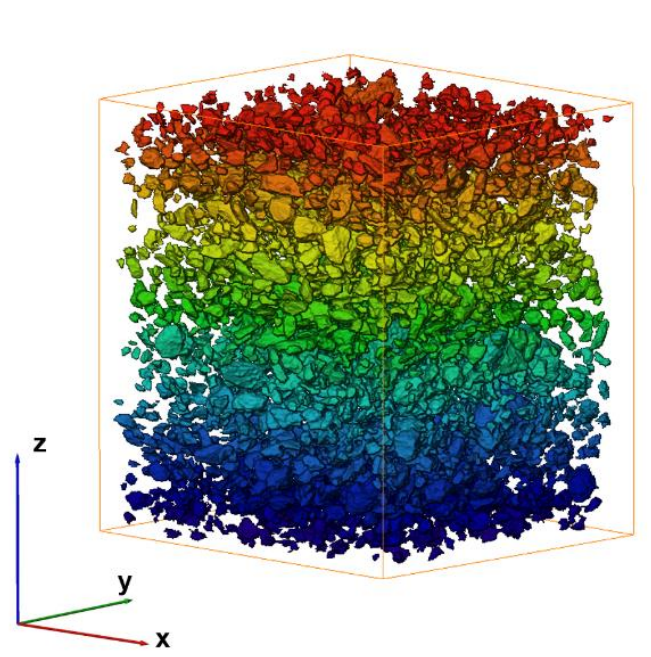

(a)

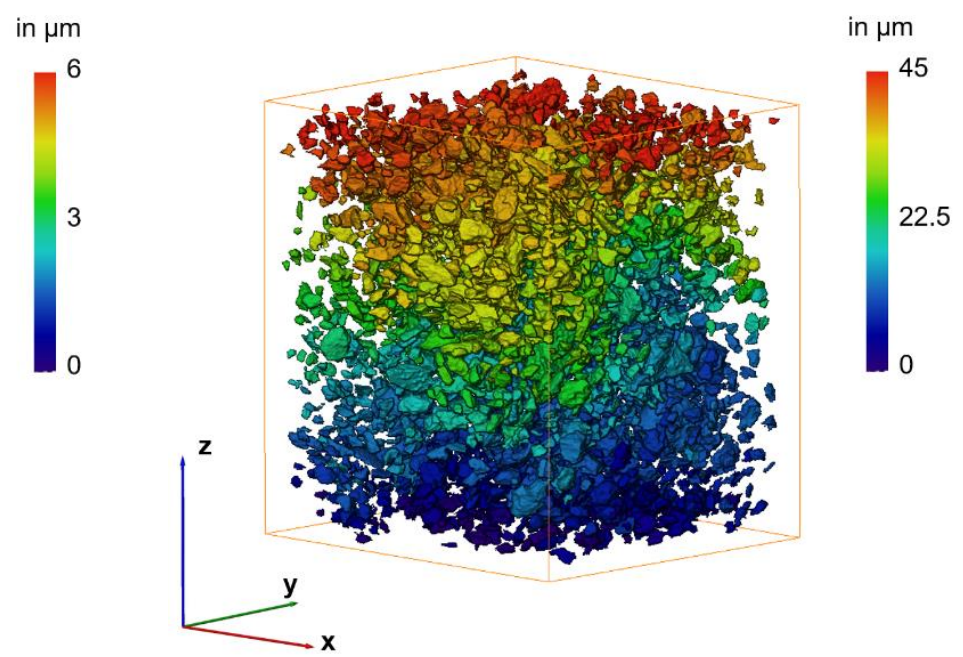

(b)

Fig. 10. Vertical displacement fields of the particles in (a) loaded state and (b) flooded (collapsed) state

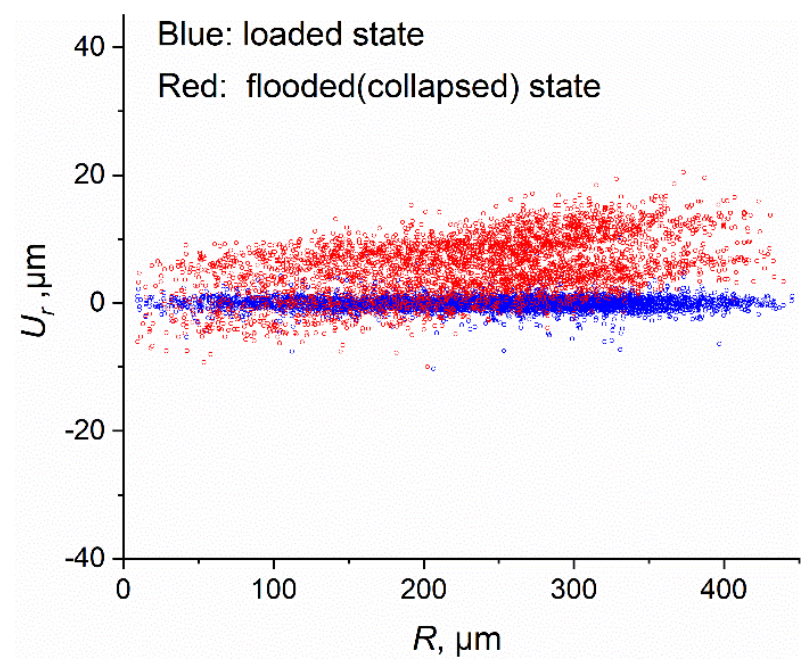

(a)

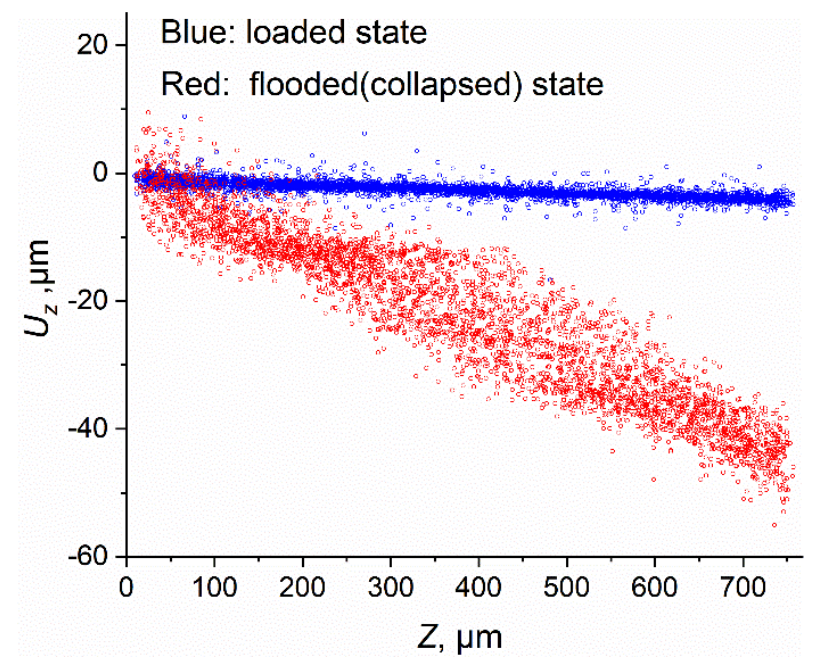

(b)

Fig. 11. Components of the particle displacements along different directions: (a) along radial axis and (b) along axial axis 


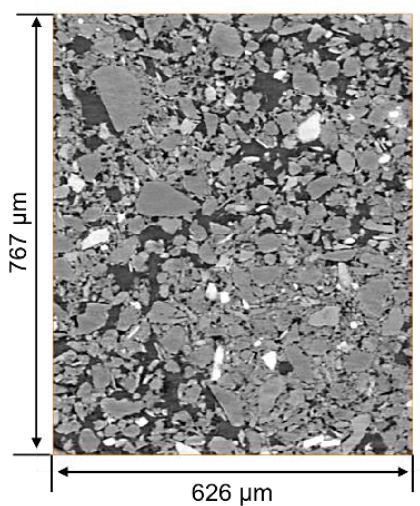

(a)

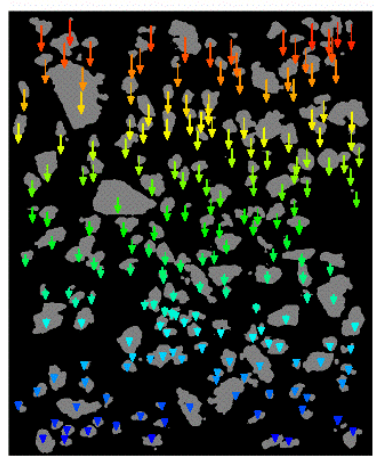

(d)

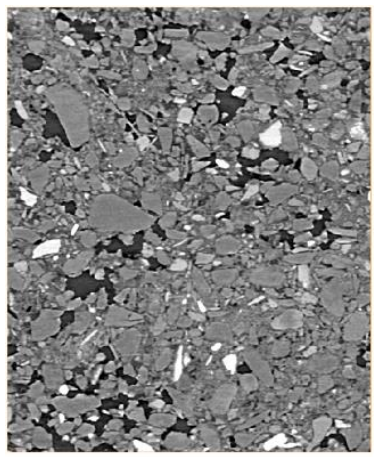

(b)

in $\mu \mathrm{m}$

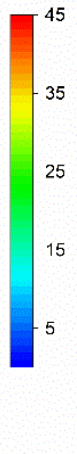

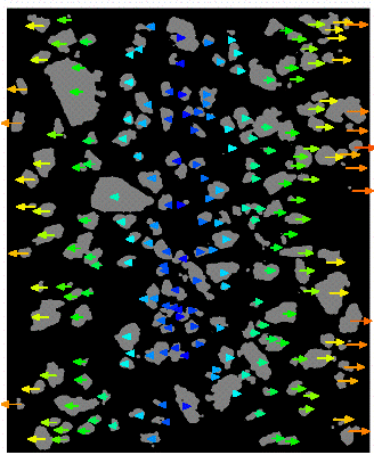

(e)

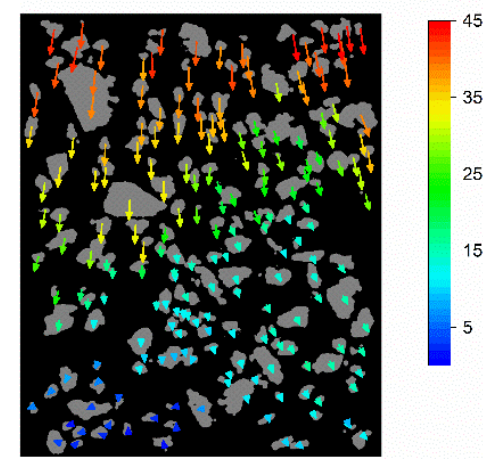

(c)

in $\mu \mathrm{m}$
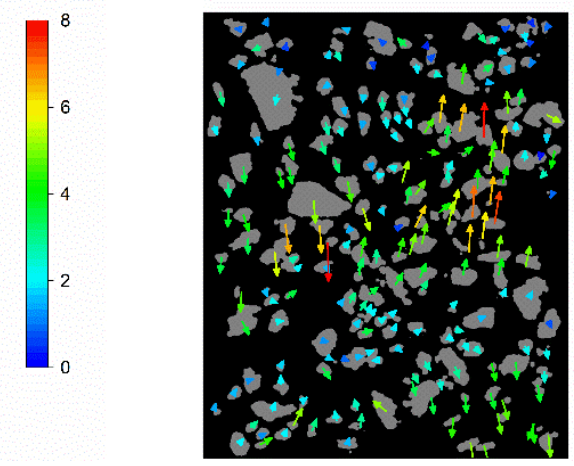

(f)

Fig. 12. (a) and (b): Selected slices in the same vertical plane extracted from the tomography image volumes in initial and flooded (collapsed) states; (c) total displacement vectors plotted on identified particles within the plane; (d) and (e) global displacement vectors along axial and radial axis; (f) local displacement vectors 


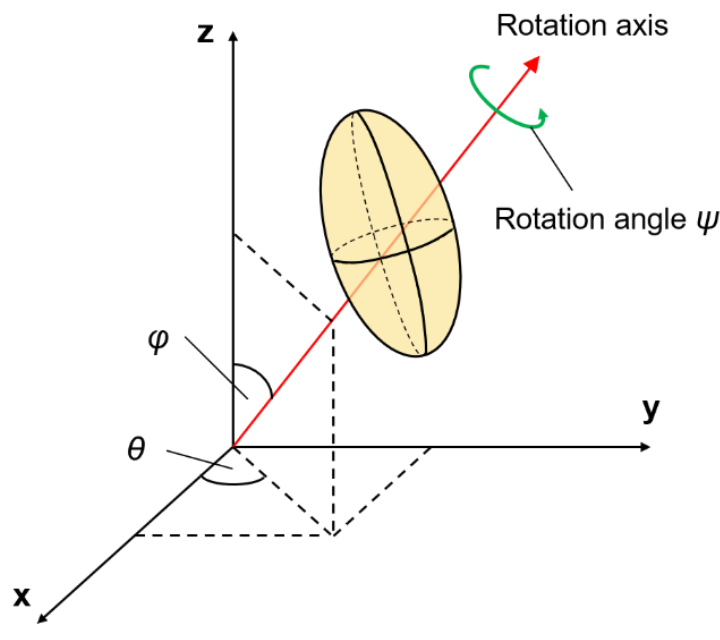

(a)

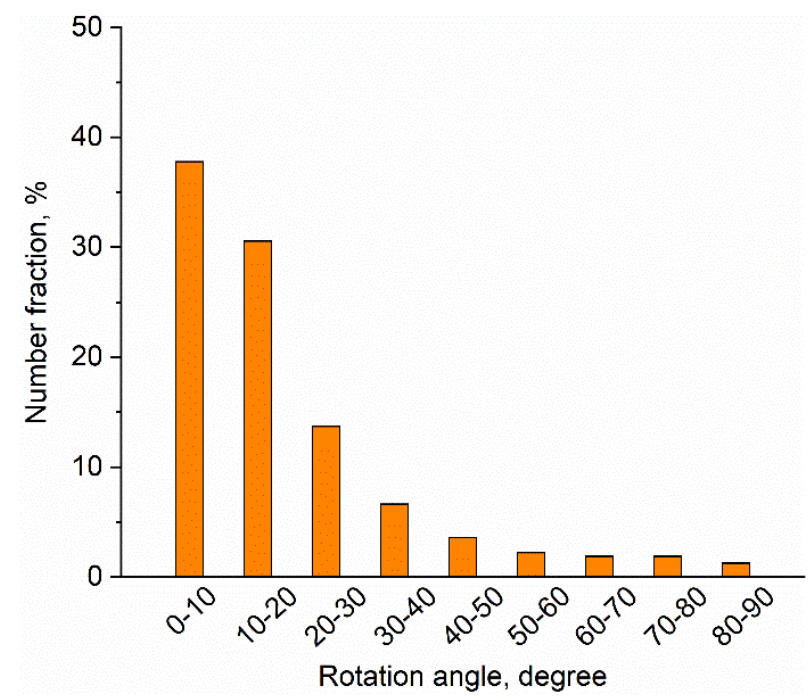

(c)

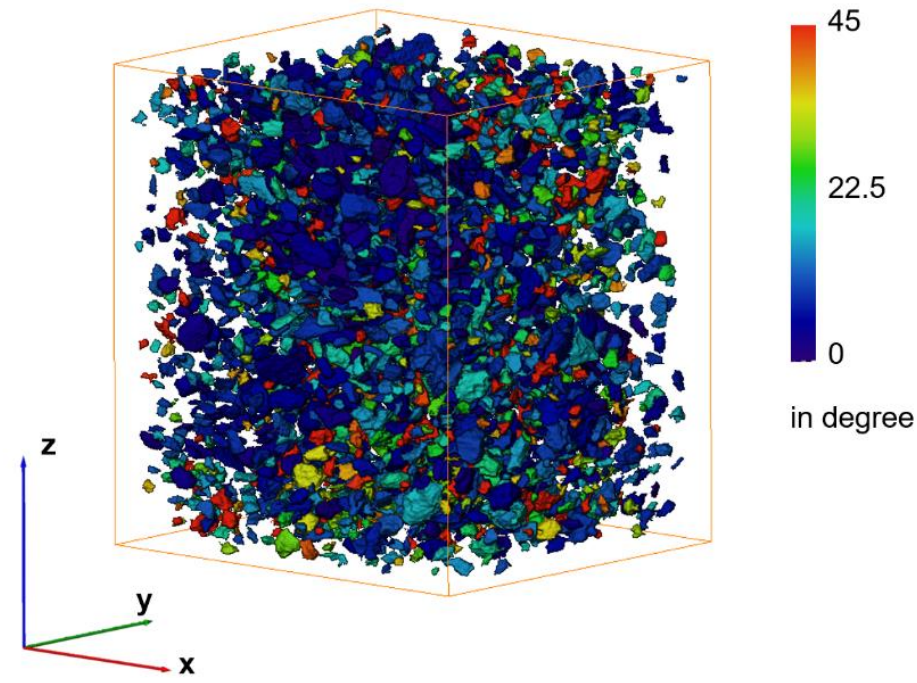

(b)

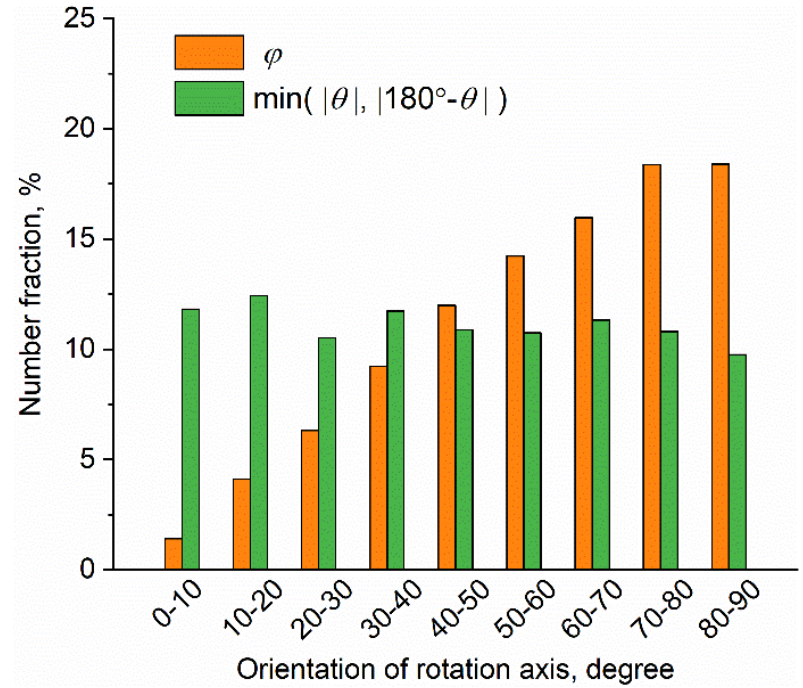

(d)

Fig. 13. (a) Illustration of the horizontal coordinate system used to describe particle rotations; (b) magnitude of particle rotation angle in the collapsed state; (c) number fractions of particles having different rotation angles; (d) number fractions of particles having different rotation axis orientations 


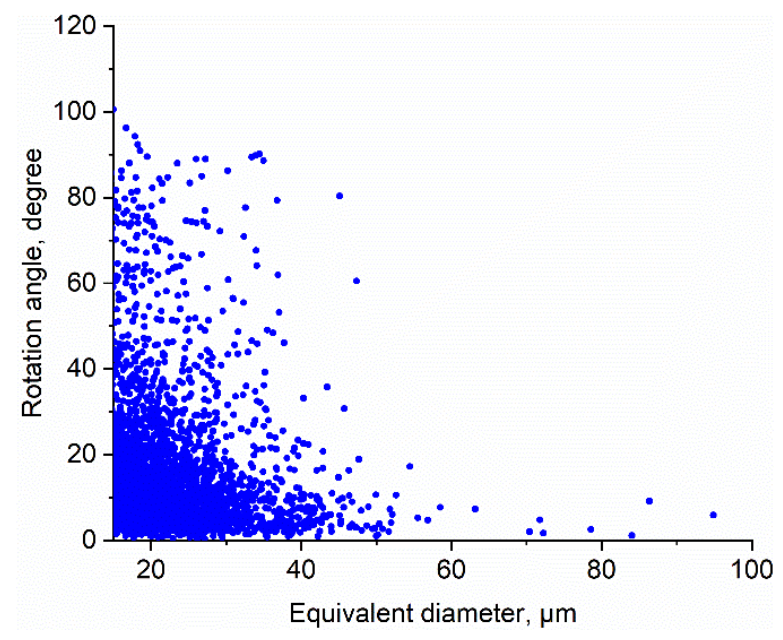

(a)

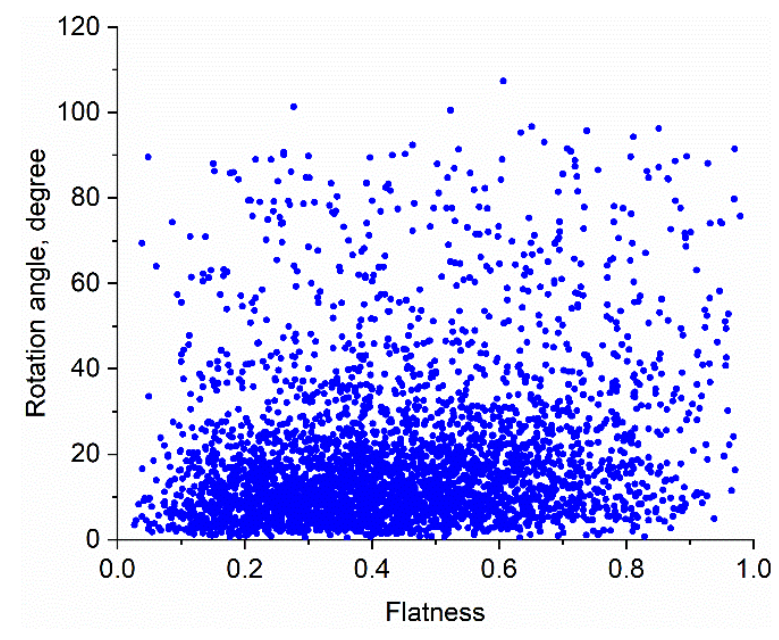

(b)

Fig. 14. Dependence of particle rotation angle on (a) particle equivalent diameter and (b) particle flatness

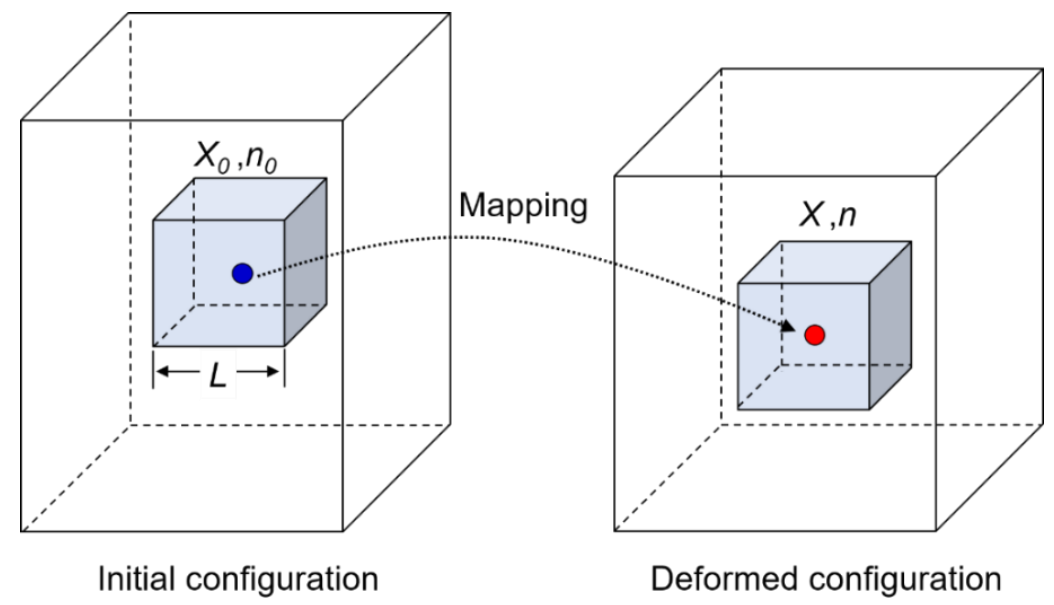

Fig. 15. Illustration of local porosity calculation and point mapping between initial and deformed configurations 


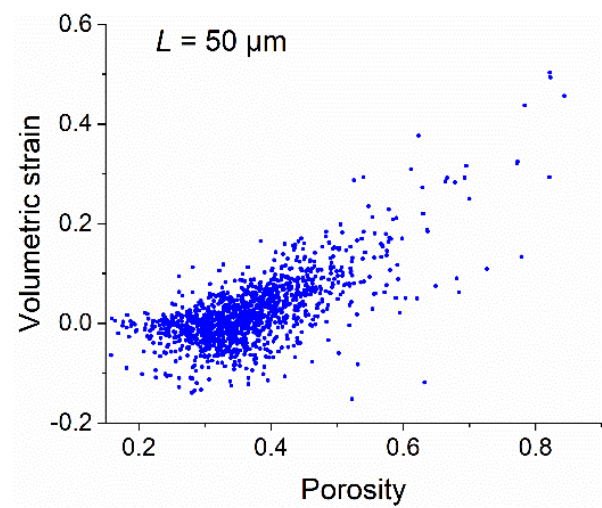

(a)

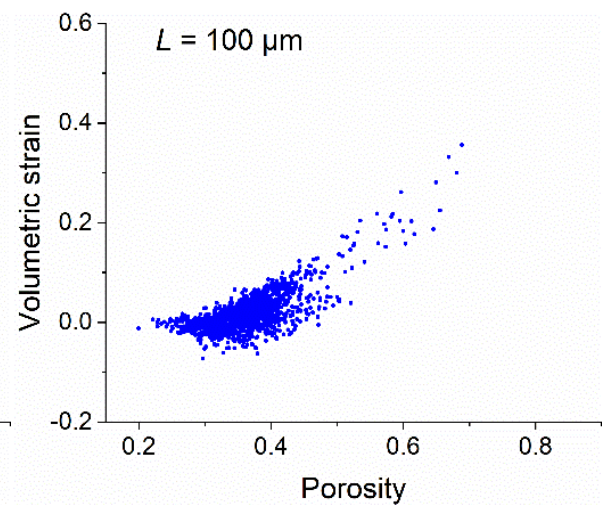

(b)

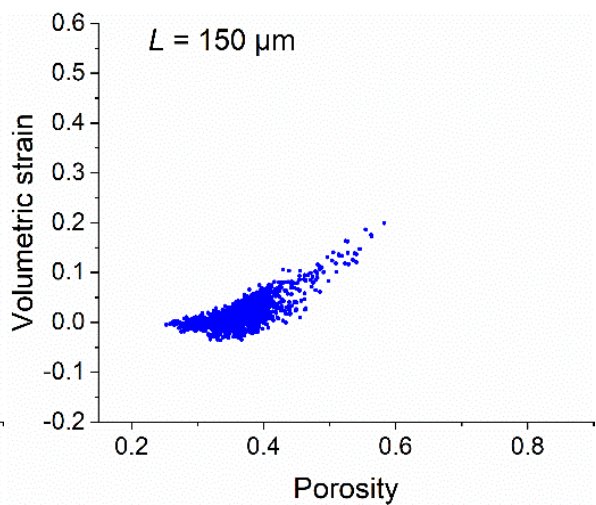

(c)

Fig. 16. Relationship between local volumetric strains and local porosities at different scales: (a) $L=50 \mu \mathrm{m}$;

(b) $L=100 \mu \mathrm{m}$; (c) $L=150 \mu \mathrm{m}$ 
Magnitude, $\mu \mathrm{m}$

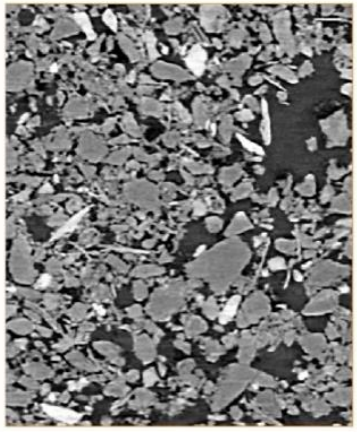

(a)
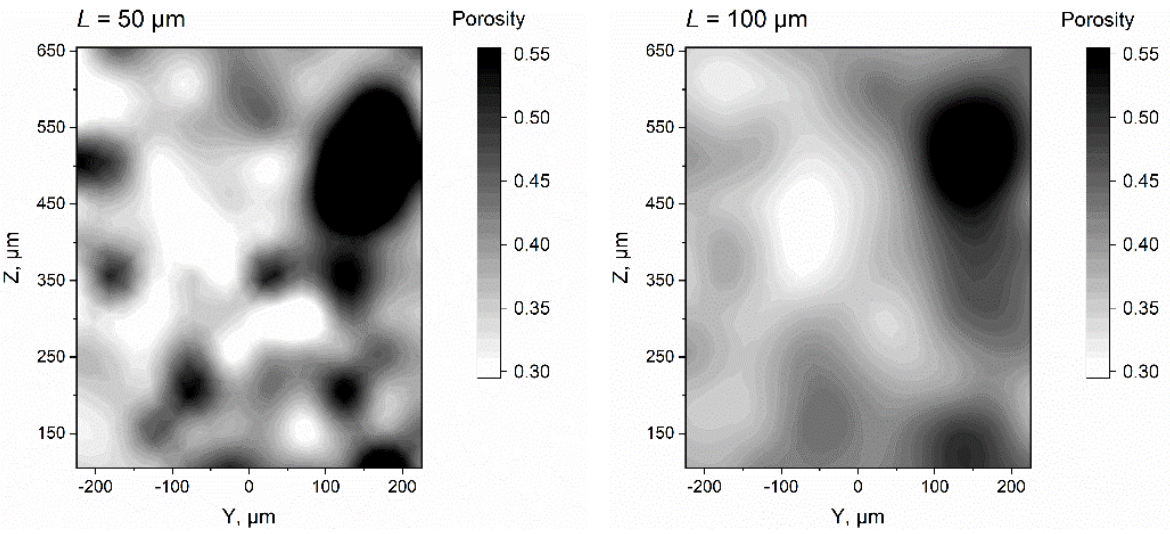

(d)
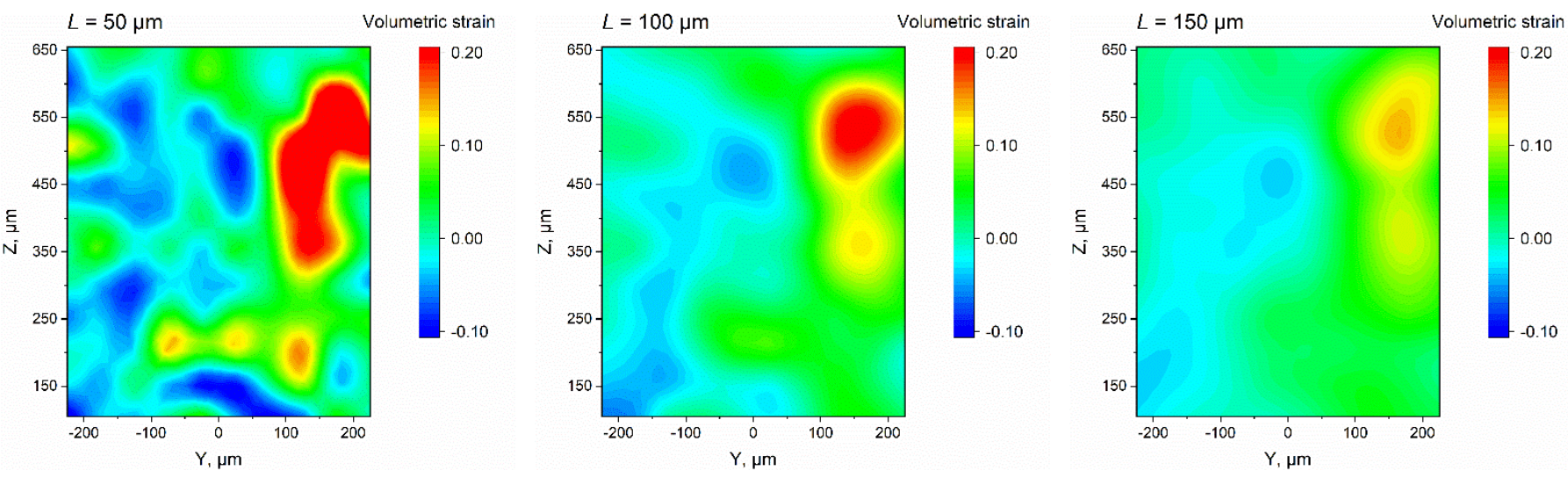

(e)

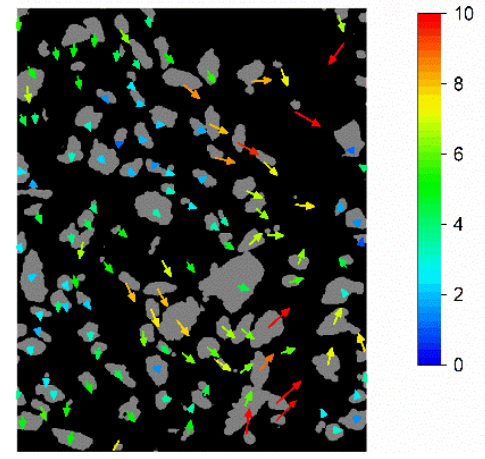

(c)

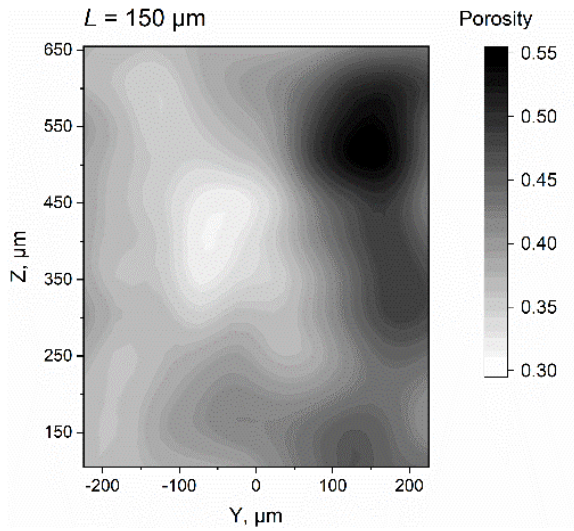
. 


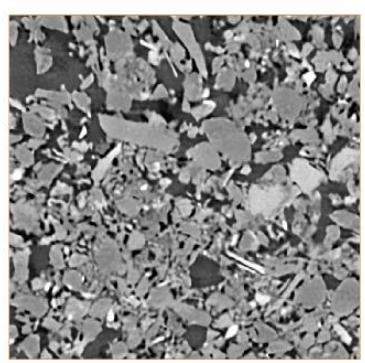

(a)

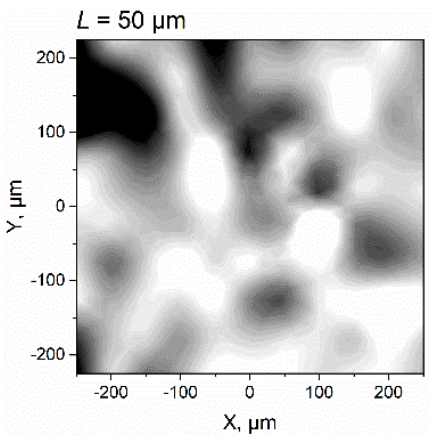

Porosity
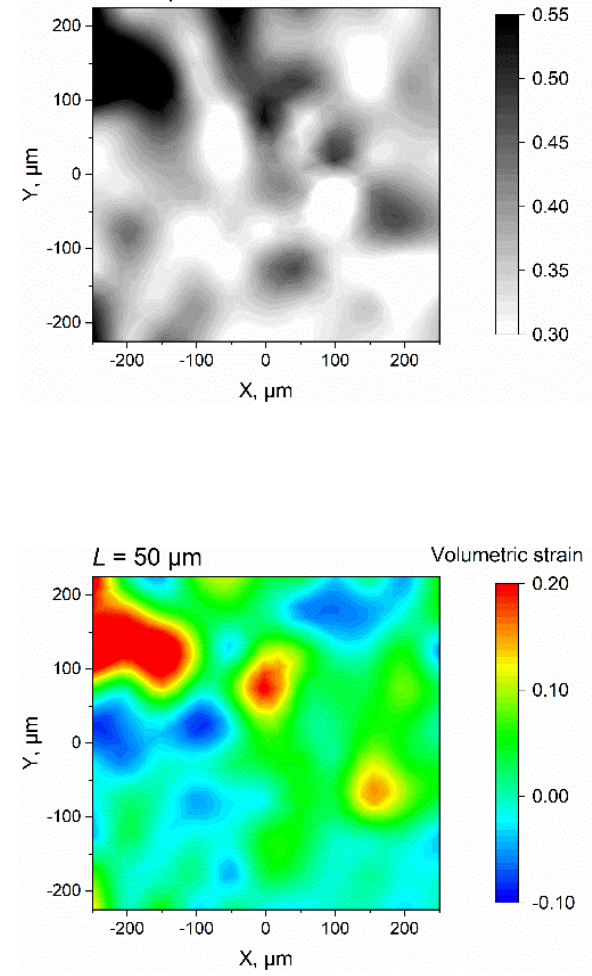

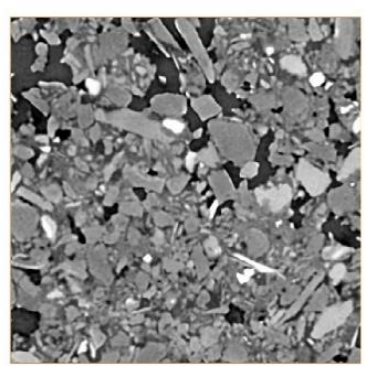

(b)
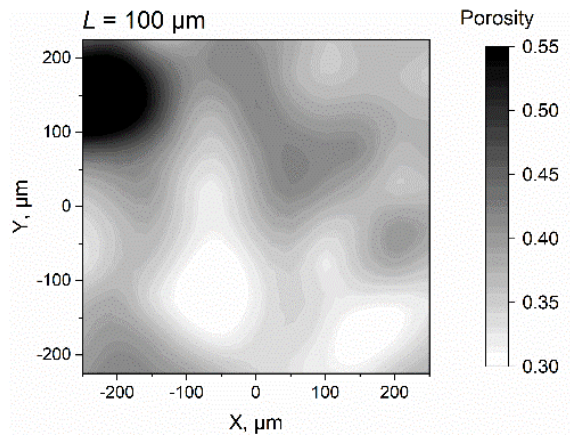

(d)
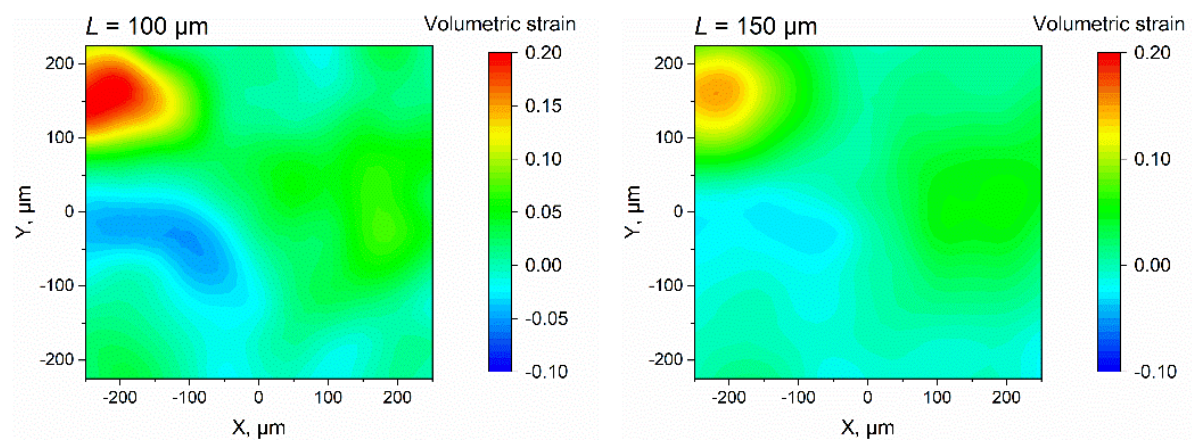

(e)

Fig. 18. For a selected slice in horizontal plane: (a) and (b), tomography images of the loess sample in initial and collapsed states; (c) local displacement vectors plotted on identified particles (vector length scaled by a factor of 5); (d) porosity and (e) volumetric strain contours at different scales 


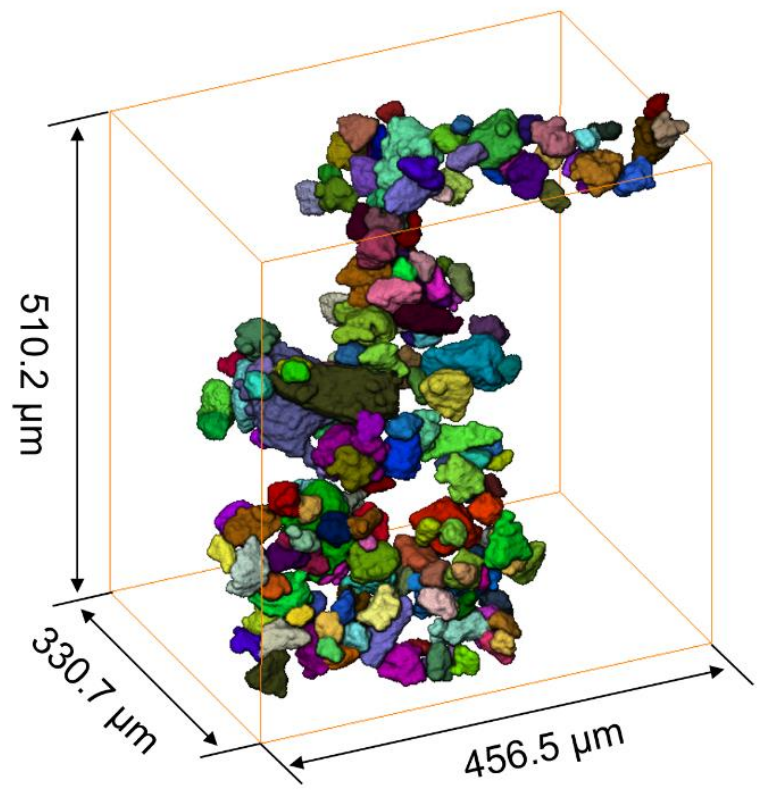

(a)

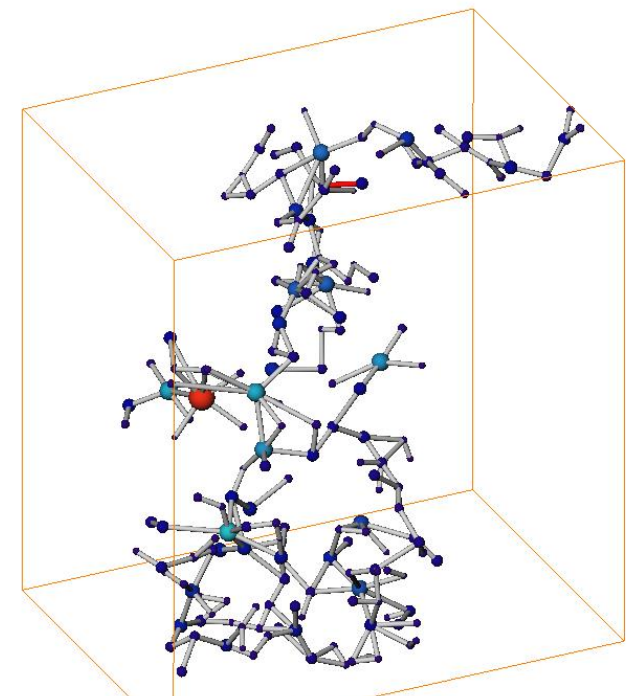

(c)

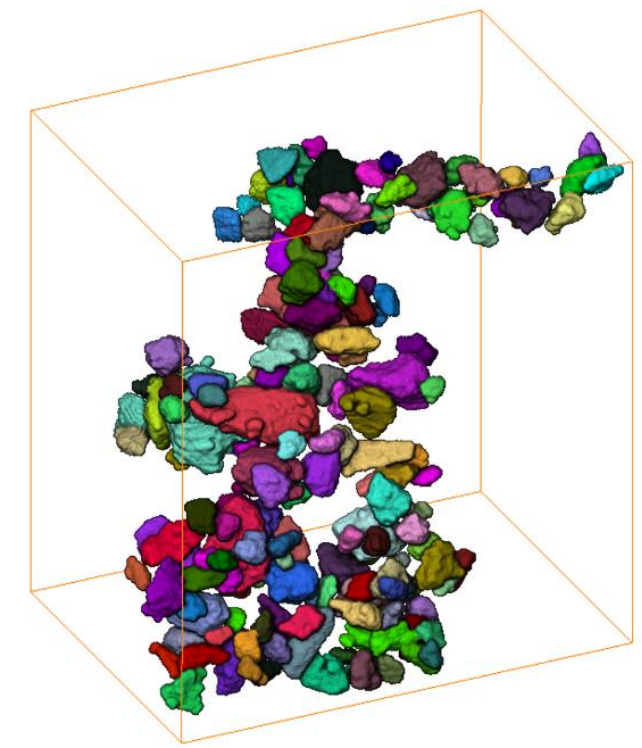

(b)

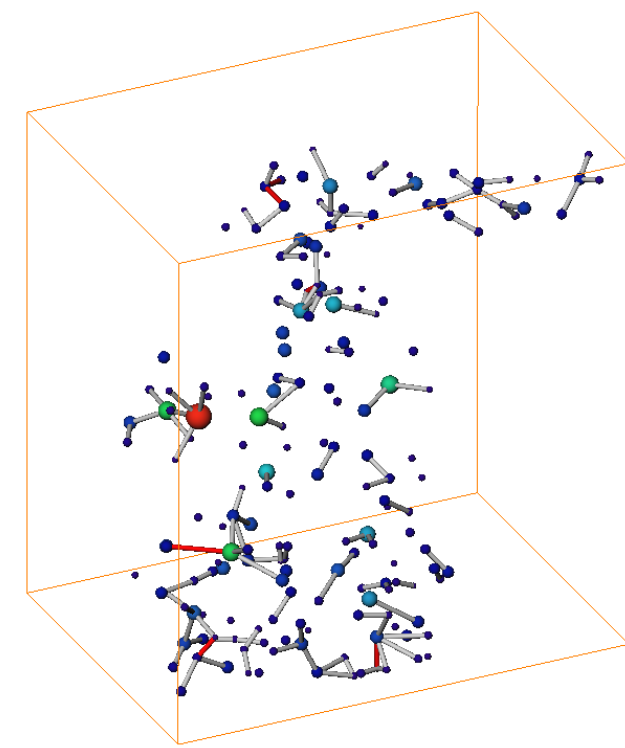

(d)

Fig. 19. Contact evolution of cluster A: (a) and (b), constituting particles in initial and collapsed states; (c) and (d), branch vector diagrams in initial and collapsed states (end point sizes scaled with the equivalent diameters by a factor 0.15 , newly formed vectors are marked red) 


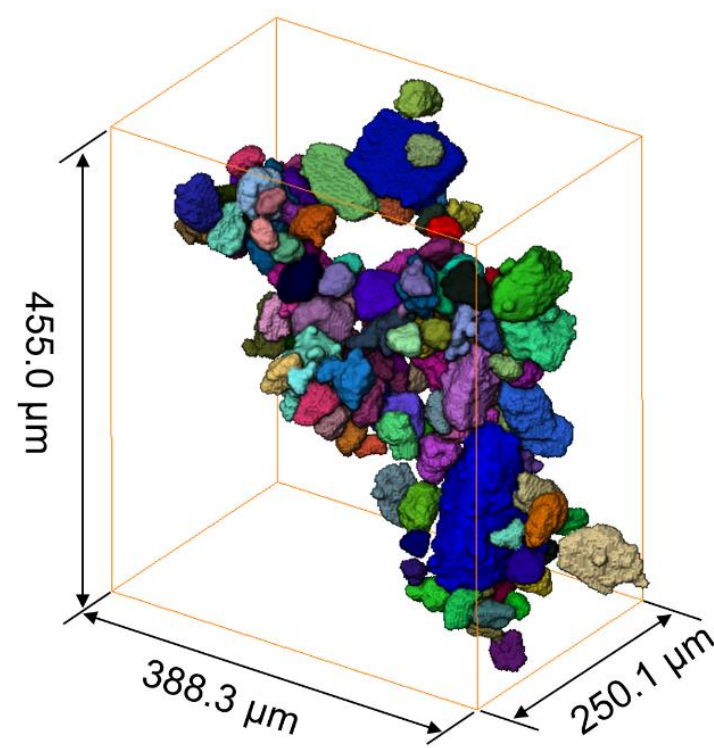

(a)

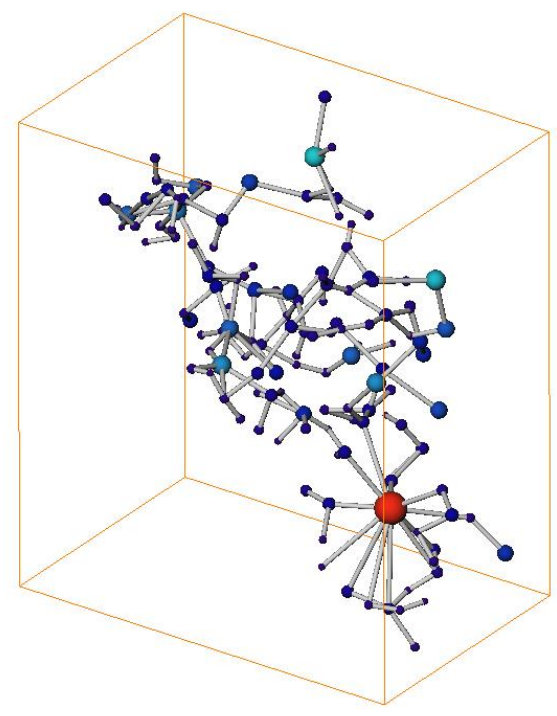

(c)

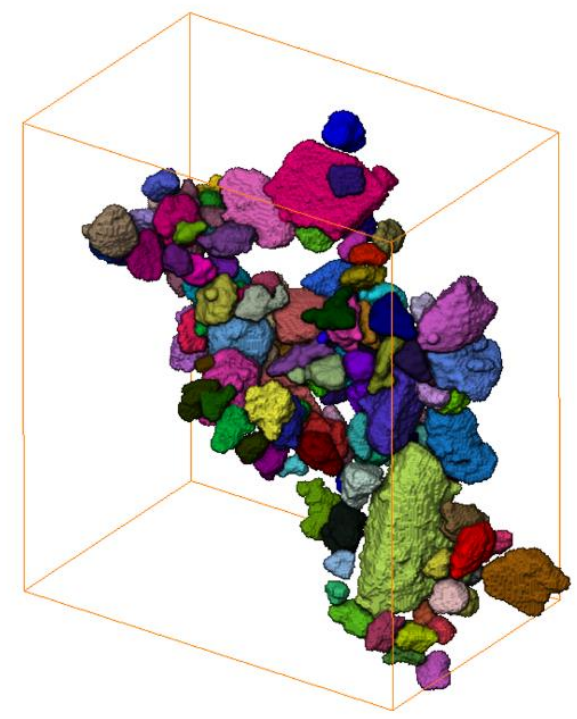

(b)

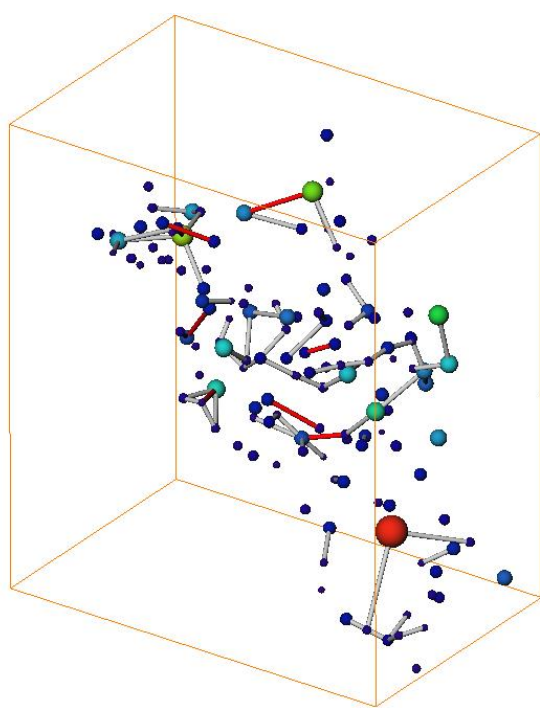

(d)

Fig. 20. Contact evolution of cluster B: (a) and (b), constituting particles in initial and collapsed states; (c) and (d), branch vector diagrams in initial and collapsed states (end point sizes scaled with the equivalent diameters by a factor 0.15 , newly formed vectors are marked red) 